\title{
An elliptic theory of indicial weights and applications to non-linear geometry problems
}

\author{
Yuanqi Wang*
}

\begin{abstract}
Given an elliptic operator $P$ on a non-compact manifold (with proper asymptotic conditions), there is a discrete set of numbers called indicial roots. It's known that $P$ is Fredholm between weighted Sobolev spaces if and only if the weight is not indicial. We show that an elliptic theory exists even when the weight is indicial. We also discuss some simple applications to Yang-Mills theory and minimal surfaces.
\end{abstract}

\section{Introduction}

\subsection{The theory}

The elliptic theories based on weighted Sobolev (Schauder) spaces usually concern a discrete set of real numbers. If a number is in the set, we say that it is indicial (or is an indicial root). A classical fact says that on a non-compact complete manifold, an elliptic operator (with proper asymptotic conditions) is Fredholm between weighted Sobolev spaces if and only if the weight is not indicial. For earlier pioneering work, please see [10], 11], and [14. For more recent work, please see [12.

Following elementary ideas, we show that there is an elliptic theory even if the weight is indicial: first, we add polynomial weights $\{$ compare (6) to [11, (1.3)]\} to refine the space; second, we consider graph norms with respect to the model operator [see (2)].

In this note we only consider first and second-order operators modelled on the following.

Definition 1.1. Let $Y$ be a $(n-1)$-dimensional Riemannian manifold without boundary (which does not have to be connected). Let $E, F$ be smooth vector-bundles over $Y$ equipped with smooth Hermitian metrics. Given arbitrary bundle isomorphisms $\sigma_{1}: E \rightarrow F, \sigma_{2}$ : $E \rightarrow E$, we say that an operator $P^{0}$ is TID (translation-invariant and diagonal) if

$$
P^{0}=\sigma_{1}\left(-B_{P^{0}}-a_{1} \frac{\partial}{\partial t}+a_{2} \frac{\partial^{2}}{\partial t^{2}}\right) \sigma_{2} \text { and the following holds. }
$$

- $a_{2}=0$ or 1 . $a_{1}=-1$ when $a_{2}=0$ (always achievable by normalization).

- When $a_{2}=0, B_{P^{0}}$ is a first-order self-adjoint elliptic differential operator $C^{\infty}(Y, E) \rightarrow$ $C^{\infty}(Y, E)$. When $a_{2}=1, B_{P^{0}}$ is second-order, simple, elliptic, and self-adjoint $C^{\infty}(Y, E) \rightarrow C^{\infty}(Y, E)$ (see Definition 2.1).

Remark 1.2. For any TID operator $P^{0}, S p e c B_{P^{0}}$ is real and discrete. Moreover, there is a complete eigen-basis of $B_{P^{0}}$.

Definition 1.3. Let $P^{0}$ be TID, and $(\beta, \Lambda)$ be a pair of real numbers such that $\Lambda \in$ $\operatorname{Spec}\left(B_{P^{0}}\right)$. When $P^{0}$ is first-order, we say that $(\beta, \Lambda)$ is $P^{0}$-indicial if $\beta=\Lambda$. When $P^{0}$ is second-order, we say that $(\beta, \Lambda)$ is $P^{0}$-indicial if

1. $\beta \neq \frac{a_{1}}{2}$ and $\Lambda-\beta^{2}+a_{1} \beta=0$, or if

2. $\Lambda \leq-\frac{a_{1}^{2}}{4}$ and $\beta=\frac{a_{1}}{2}$.

\footnotetext{
*Department of Mathematics, Stony Brook University, NY, USA. ywang@scgp.stonybrook.edu.
} 
In the second case above, we say that $(\beta, \Lambda)$ is $P^{0}$-super indicial. We say that $\beta$ is $P^{0}$-indicial (super indicial) if there is a $\Lambda \in \operatorname{Spec}_{P^{0}}$ such that $(\beta, \Lambda)$ is $P^{0}$-indicial (super indicial). This is consistent with the " $\mathfrak{D}_{A}$ " in [11, page 417], translated to our setting.

Let $N$ be a complete Riemannian manifold with finite many cylindrical ends, we consider asymptotically TID operators $P: C^{\infty}(N, E) \rightarrow C^{\infty}(N, F)$. This class should include most of the Dirac and Laplace-type operators in geometry. In the setting as Theorem 1.4.

$$
\begin{array}{ll}
P: \widehat{W}_{-\beta, \gamma, b-1}^{k+m_{0}, p}(N, E) \longrightarrow W_{-\beta, \gamma, b}^{k, p}(N, F) & \left(\left.\square\right|_{-\beta, \gamma, b} ^{\text {Sobolev }, p}\right) \text { (see Definition } 2.3 \\
P: \widehat{C}_{-\beta, \gamma, b-1}^{k+m_{0}, \alpha}(N, E) \longrightarrow C_{-\beta, \gamma, b}^{k, \alpha}(N, F) & \left(\left.\square\right|_{-\beta, \gamma, b} ^{\text {Schauder }}\right) \quad \text { for the norms). }
\end{array}
$$

are bounded operators. Moreover, when $\beta$ is not indicial and $\gamma=0$, as subspaces of $L_{l o c}^{2}$,

$$
\begin{gathered}
\widehat{W}_{-\beta, \gamma, b-1}^{k+m_{0}, p}(N, E)=W_{-\beta}^{k+m_{0}, p}(N, E), \widehat{C}_{-\beta, \gamma, b-1}^{k+m_{0}, \alpha}(N, E)=C_{-\beta}^{k+m_{0}, \alpha}(N, E), \\
W_{-\beta, \gamma, b}^{k, p}(N, F)=W_{-\beta}^{k, p}(N, F), C_{-\beta, \gamma, b}^{k, \alpha}(N, F)=C_{-\beta}^{k, \alpha}(N, F) .
\end{gathered}
$$

Thus our theory generalizes the one in [11] \{for first and second-order operators, c.f [11, (1.3)]\}. Assuming the weights are the same on the ends, our main result states as follows.

Theorem 1.4. Suppose $P$ is a $\beta-$ ATID elliptic operator (see Definition 2.8), and $\beta$ is not $P^{0}$-super indicial. Then for any $0 \leq k \leq k_{0}-2, \alpha \in(0,1), p \geq 2$,

- $\left(\left.\square\right|_{-\beta, \gamma, b} ^{S o b o l e v, p}\right)$ is Fredholm if $b \neq 1-\frac{1}{p}$ or $\beta$ is not $P^{0}$ - indicial;

- $\left(\square||_{-\beta, \gamma, b}^{\text {Schauder }}\right)$ is Fredholm if $b \neq 1$ or $\beta$ is not $P^{0}$ - indicial.

Remark 1.5. The super-indicial roots are essentially different from the ordinary ones. Fortunately, they don't exist for first-order operators, and they barely appear on second-order operators. For example, any super-indicial root in the setting of Corollary 1.9 must be positive, but we only need $\beta$ to be non-positive therein.

Remark 1.6. Theorem 5.2 and Proposition 4.5 give reasonably general index formulas for first-order operators (see Remark 5.3). As a by-product, we prove an obvious identity (Proposition 5.4) on the eta-invariant defined in 3. It can also be proved by the Fredholm theory in [3. However, the author is not able to find Proposition 5.4 in the literature.

Remark 1.7. Though the indicial roots do not prevent Fredholmnness, the index still changes when $\beta$ goes across any of them (c.f. [11, Last 5 lines in Page 433]). 2.11).

Our theory still works when the weights are not the same on the ends (see Theorem

Computations indicate that the our local inverses (Theorem 3.2 ) are different from those of Lockhart-McOwen [11, (2.3)] (by Fourier-transform in the $t$-direction). When $k<0$, our local inverses do not work for the $W^{k, p}\left(C^{k, \alpha}\right)$ theories.

Remark 1.8. Assuming that $P$ is translation invariant on each end, Theorem 1.4, 2.11 are still true with "if" replaced by "if and only if" (see the proof in the Appendix). However, only assuming $\beta$-ATID, when $b=1$, we don't know whether $\left(\square \mid \begin{array}{l}\text { Schauder } \\ -\beta, \gamma, b\end{array}\right)$ is not Fredholm. The same doubt applies to the Sobolev theory.

By simple conformal changes as in [11, Section 9], Theorem 1.4 is equivalent to a theory in the conic setting (and hopefully the asymptotic conic setting). Please also see [15] and the discussion above Lemma 7.2 .

Under stronger asymptotic conditions than Definition 2.8, we have a theory for superindicial roots (on second-order operators), and a theory for powers of Laplace-type operators i.e. $\Delta^{m},(m \geq 2)$. The higher-order operators include (linearisation of) the extremal metric operator in [4], and the conformal co-variant operators in [5]. We will address these in the future when geometric motivation arises.

Amrouch-Girault-Giroire [2] also use Sobolev-spaces with polynomial weights to study Laplace equations on domains. It's possible that our theory is essentially similar to theirs. 


\subsection{Simple applications}

Geometric objects with isolated conic singularities usually converge to their tangent cones polynomially (see [16]). Let $r$ be the distance to the singular point, and $t=-\log r$ be the cylindrical coordinate. Our work implies a general phenomenon: the rate of convergence to the tangent cone is either exponential or not faster than $\frac{1}{t}$ ( or $\left.\frac{1}{-\log r}\right)$.

We first do minimal sub-manifolds. In the cylindrical setting, we say that a minimal graph sub-manifold is asymptotic to a cone at a certain rate, if the section " $u$ " in (63) converges to 0 at the rate (see Definition 6.1).

Corollary 1.9. Suppose $\Sigma$ is a n-dimensional closed minimal sub-manifold in $\mathbb{S}^{N}, n \geq 1$. Let $\underline{0}$ be the negative number in Definition 3.1 with respect to the $L_{\Sigma}$ in (63). Then there is $a \delta_{0}$ depending on $\Sigma$ with the following property.

Suppose $\widehat{\Sigma}$ is a (locally defined) embedded minimal sub-manifold in $\mathbb{R}^{N+1}$ with isolated cone singularity at $O$. Suppose $\widehat{\Sigma}$ is a graph over Cone $(\Sigma)$, and in the cylindrical setting, it converges to Cone $(\Sigma)$ at least at the rate $\frac{\delta_{0}}{t}$ (see Definition [6.1). Then $\widehat{\Sigma}$ converges to Cone $(\Sigma)$ exponentially at the rate $O\left(e^{-|\underline{0}| t}\right)$.

Remark 1.10. By Definition 2.8 and Remark 2.9, we can not make $\delta_{0}$ small by scaling. Adam-Simon 1 showed that there are singular minimal sub-manifolds converging to a cone at a rate comparable to $(-\log r)^{-1}$. This suggests that in general, the assumption on the rate in Corollary 1.9 can not be weaken.

Similar results hold for Yang-Mills connections as well.

Corollary 1.11. Let $n \geq 5$. Suppose $g$ is a smooth metric on $B_{O}^{n}(R)$ and $g(O)=g_{E}$ (the Euclidean metric). Suppose $A_{O}$ is a $U(m)$ or $S O(m)$ Yang-Mills connection on (the unit round) $\mathbb{S}^{n-1}$. Let $\underline{0}$ be the negative number in Definition 3.1 with respect to the $B$ in (60). Then there is a $\delta_{0}>0$ depending on $A_{O}$ with the following properties.

Suppose $A$ is a smooth Yang-Mills connection on $B_{O}(R) \backslash O$. In the cylindrical setting as Section 6.1, suppose $A$ converges to Cone $\left(A_{O}\right)$ at least at the rate $\frac{\delta_{0}}{t}$ (see Definition 6.1).

$I$ : Suppose $A$ is in Coulomb gauge relative to $A_{O}$ (with respect to $g$ or the Euclidean metric). Then $A$ converges to Cone $\left(A_{O}\right)$ exponentially at the following rate.

$$
\left\{\begin{array}{cl}
O\left(e^{-|\underline{0}| t}\right) & \text { when }|\underline{0}|<1, \\
O\left(e^{-t}\right) & \text { when }|\underline{0}|>1 \\
O\left(t e^{-|\underline{0}| t}\right) & \text { when }|\underline{0}|=1
\end{array}\right.
$$

$I I$ : When $A_{O}$ is irreducible, there exists a gauge s such that $s(A)$ converges to $C o n e\left(A_{O}\right)$ exponentially as (4).

Remark 1.12. By (59), when $n=4$, the weight 0 is super-indicial unless $B$ is positively definite.

Organization of this note: the norms can be found in Section 2 , We construct the local inverses in Section 3. In Section 4, we study regularity of harmonic sections, and complete the proof for Theorem 1.4. We give the index formula (for first-order operators) and study the eta-invariant in Section [5. We prove Corollary [1.9, 1.11 in Section 6.

Acknowledgement: The author would like to thank Professor Simon Donaldson, Thomas Walpuski, and Lorenzo Foscolo for helpful discussions.

\section{Preparation}

Definition 2.1. In the setting of Definition 1.1, we say that an operator $H: C^{\infty}(Y, E) \rightarrow$ $C^{\infty}(Y, E)$ is admissible, if there is a linear first-order differential operator $H_{0}$, and sections $\widehat{\Gamma}_{k}, \Gamma_{k} \in C^{\infty}(Y, E)$ such that

$$
H \xi=H_{0} \xi+\Sigma_{k=1}^{k_{0}} \widehat{\Gamma}_{k} \int_{Y}<\xi, \Gamma_{k}>d V .
$$


We say that a second-order operator $B: C^{\infty}(Y, E) \rightarrow C^{\infty}(Y, E)$ is simple, if there is a smooth connection $A_{0}$ on $E$ and a smooth metric $\widehat{g}$ on $Y$ (which does not need to be the given one), such that $B-\nabla_{A_{0}}^{\star \widehat{g}} \nabla_{A_{0}}$ is admissible.

Definition 2.2. (Strips) Let $k S_{m}=(m-k, m+k)$, and $\left\{\xi_{m}\right\}_{m=3}^{\infty}$ be a partition of unity of $C y l_{1}$ subordinate to the cover $\left\{2 S_{m}\right\}_{m=3}^{\infty}$ i.e. $\xi_{m}$ is supported in $2 S_{m}$ and is $\equiv 1$ in $S_{m}$.

Definition 2.3. Let $C y l_{t_{0}}$ denote $Y \times\left(t_{0}, \infty\right), t_{0} \geq 0.1$, we define the $L_{-\beta, b}^{2}\left[C y l_{t_{0}}\right]-$ space of sections to the underlying bundle by the norm

$$
|\xi|_{L_{-\beta, b}^{p}\left(C y l_{t_{0}}\right)} \triangleq\left|e^{-\beta t} t^{b} \xi\right|_{L^{p}\left(C y l_{t_{0}}\right)}=\left(\int_{C y l_{t_{0}}}\left|e^{-\beta t} t^{b} \xi\right|^{p}\right)^{\frac{1}{p}} .
$$

We define $|\xi|_{W_{-\beta, b}^{k, p}\left(C y l_{t_{0}}\right)} \triangleq \Sigma_{j=0}^{k}\left|\nabla_{A_{0}}^{j} \xi\right|_{L_{-\beta, b}^{p}\left(C y l_{t_{0}}\right)}$. For the Schauder theory, we define $|\xi|_{C_{-\beta, b}^{k, \alpha}\left(\overline{C y l}_{t_{0}}\right)} \triangleq \sup _{m \geq t_{0}+1} m^{b} e^{-\beta m}|\xi|_{C^{k, \alpha}\left(S_{m}\right)}$.

Let $\xi^{\|_{\beta}}$ denote the projection of $\xi$ onto $\operatorname{Ker}\left\{B_{P^{0}}-\beta I d\right\}$ (for all $t$ ), and $\xi^{\perp_{\beta}}=\xi-\xi^{\|_{\beta}}$ be the perpendicular vector. When $P^{0}$ is first-order, we define

$$
\begin{aligned}
& \left|\sigma_{2}^{-1} \xi\right|_{\widehat{W}_{-\beta, \gamma, b-1}^{k, p, P^{0}}\left(C y l_{t_{0}}\right)} \triangleq\left|\xi^{\perp_{\beta}}\right|_{W_{-\beta, \gamma}^{k, p}\left(C y l_{t_{0}}\right)}+\left|\xi^{\|_{\beta}}\right|_{W_{-\beta, b-1}^{k, p}\left(C y l_{t_{0}}\right)}+\left|\frac{\partial \xi^{\|_{\beta}}}{\partial t}-\beta \xi^{\|_{\beta}}\right|_{W_{-\beta, b}^{k-1, p}\left(C y l_{t_{0}}\right)} \\
& \left|\sigma_{2}^{-1} \xi\right|_{W_{-\beta, \gamma, b}^{k, p, P}\left(C y l_{t_{0}}\right)} \triangleq\left|\xi^{\perp_{\beta}}\right|_{W_{-\beta, \gamma}^{k, p}\left(C y l_{t_{0}}\right)}+\left|\xi^{\|_{\beta}}\right|_{W_{-\beta, b}^{k, p}\left(C y l_{t_{0}}\right)} \\
& \left|\sigma_{2}^{-1} \xi\right|_{\widehat{C}_{-\beta, \gamma, b-1}^{k, \alpha, P^{0}}\left(\overline{C y l}_{t_{0}}\right)} \triangleq\left|\xi^{\perp_{\beta}}\right|_{C_{-\beta, \gamma}^{k, \alpha}\left(\overline{C y l}_{t_{0}}\right)}+\left|\xi^{\|_{\beta}}\right|_{C_{-\beta, b-1}^{k, \alpha}\left(\overline{C y l}_{t_{0}}\right)}+\left|\frac{\partial \xi^{\|_{\beta}}}{\partial t}-\beta \xi^{\|_{\beta}}\right|_{C_{-\beta, b}^{k-1, \alpha}\left(\overline{C y l}_{t_{0}}\right)} \text {. } \\
& \left.\left|\sigma_{2}^{-1} \xi\right|_{C_{-\beta, \gamma, b}^{k, \alpha}(\overline{C y l}}\right) \triangleq\left|\xi^{\perp_{\beta}}\right|_{C_{-\beta, \gamma}^{k, \alpha}\left(\overline{C y l}_{t_{0}}\right)}+\left|\xi^{\|_{\beta}}\right|_{C_{-\beta, b}^{k, \alpha}\left(\overline{C y l}_{t_{0}}\right)} \text {. }
\end{aligned}
$$

When $P^{0}$ is second-order elliptic, let $\Lambda_{\beta}=\beta^{2}-a_{1} \beta$, we define

$$
\begin{aligned}
& \left.\left|\sigma_{2}^{-1} \xi\right|_{\widehat{W}_{-\beta, \gamma, b-1}^{k, p, P}\left(C y l_{t_{0}}\right)} \triangleq\left|\xi^{\perp_{\Lambda_{\beta}}}\right|_{W_{-\beta, \gamma}^{k, p}\left(C y l_{t_{0}}\right)}+\left|\xi^{\|_{\Lambda_{\beta}}}\right|_{W_{-\beta, b-1}^{k, p}\left(C y l_{t_{0}}\right)}+\left|\frac{\partial \xi^{\| \Lambda_{\beta}}}{\partial t}-\beta \xi^{\|_{\Lambda_{\beta}}}\right|_{W_{-\beta, b}^{k-1, p}\left(C y l_{t_{0}}\right.}\right) \\
& \left.+\left|\frac{\partial^{2} \xi^{\| \Lambda_{\beta}}}{\partial t^{2}}-\beta^{2} \xi^{\|_{\Lambda_{\beta}}}\right|_{W_{-\beta, b}^{k-2, p}\left(C y l_{t_{0}}\right.}\right) \\
& \left|\sigma_{2}^{-1} \xi\right|_{W_{-\beta, \gamma, b}^{k, p, P}\left(C y l_{t_{0}}\right)} \triangleq\left|\xi^{\perp_{\Lambda_{\beta}}}\right|_{W_{-\beta, \gamma}^{k, p}\left(C y l_{t_{0}}\right)}+\left|\xi^{\|_{\Lambda_{\beta}}}\right|_{W_{-\beta, b}^{k, p}\left(C y l_{t_{0}}\right)} \\
& \left|\sigma_{2}^{-1} \xi\right|_{\widehat{C}_{-\beta, \gamma, b-1}^{k, \alpha, P^{0}}\left(\overline{C y l}_{t_{0}}\right)} \triangleq\left|\xi^{\perp_{\Lambda_{\beta}}}\right|_{C_{-\beta, \gamma}^{k, \alpha}\left(\overline{C y l}_{t_{0}}\right)}+\left|\xi^{\| \Lambda_{\beta}}\right|_{C_{-\beta, b-1}^{k, \alpha}\left(\overline{C y l}_{t_{0}}\right)}+\left|\frac{\partial \xi^{\| \Lambda_{\beta}}}{\partial t}-\beta \xi^{\|_{\Lambda_{\beta}}}\right|_{C_{-\beta, b}^{k-1, \alpha}\left(\overline{C y l}_{t_{0}}\right)} \\
& +\left|\frac{\partial^{2} \xi^{\| \Lambda_{\beta}}}{\partial t^{2}}-\beta^{2} \xi^{\|_{\Lambda_{\beta}}}\right|_{C_{-\beta, b}^{k-2, \alpha}\left(\overline{C y l}_{t_{0}}\right)} \text {. } \\
& \left|\sigma_{2}^{-1} \xi\right|_{C_{-\beta, \gamma, b}^{k, \alpha, P^{0}}\left(\overline{C y l}_{t_{0}}\right)} \triangleq\left|\xi^{\perp_{\Lambda_{\beta}}}\right|_{C_{-\beta, \gamma}^{k, \alpha}\left(\overline{C y l}_{t_{0}}\right)}+\left|\xi^{\|_{\Lambda_{\beta}}}\right|_{C_{-\beta, b}^{k, \alpha}\left(\overline{C y l}_{t_{0}}\right)} .
\end{aligned}
$$

Remark 2.4. The $\sigma_{2}$ of the adjoint operator $L^{\star}$ in (52) is usually not identity, but it never affects the index or kernel.

For all first and second-order TID-operators, we abuse notation and denote the corresponding operators on the link as $B_{P^{0}}$. We need to solve the equations

$$
\left(\frac{\partial}{\partial t}-B_{P_{0}}\right) \sigma_{2} \xi=\sigma_{1}^{-1} h,\left(\frac{\partial^{2}}{\partial t^{2}}-a_{1} \frac{\partial}{\partial t}-B_{P_{0}}\right) \sigma_{2} \xi=\sigma_{1}^{-1} h \text { respectively. }
$$

Let $u=\left(\sigma_{2} \xi\right) e^{-\beta t}, f=\left(\sigma_{1}^{-1} h\right) e^{-\beta t}$, (7) become

$$
\frac{\partial u}{\partial t}-B_{P_{\beta}^{0}} u=f \text { and } \frac{\partial^{2} u}{\partial t^{2}}-\left(a_{1}-2 \beta\right) \frac{\partial u}{\partial t}-B_{P_{\beta}^{0}} u=f \text { respectively, }
$$

where

$$
B_{P_{\beta}^{0}} \triangleq\left\{\begin{array}{cc}
B_{P^{0}}-\beta I d & \text { when } P^{0} \text { is first-order } \\
B_{P^{0}}+a_{1} \beta-\beta^{2} & \text { when } P^{0} \text { is second-order elliptic. }
\end{array}\right.
$$


For any $\Lambda \in \operatorname{Spec}\left(B_{P^{0}}\right)$ (repeated by multiplicity). Let

$$
\lambda\left[\in \operatorname{Spec}\left(B_{P_{\beta}^{0}}\right)\right] \triangleq\left\{\begin{array}{cc}
\Lambda-\beta & \text { when } P^{0} \text { is first-order, } \\
\Lambda+a_{1} \beta-\beta^{2} & \text { when } P^{0} \text { is second-order elliptic. }
\end{array}\right.
$$

Let $\left[\phi_{\Lambda}, \Lambda \in \operatorname{Spec}\left(B_{P^{0}}\right)\right]$ denote the orthonormal eigen-basis of $L^{2}[Y, E]$ with respect to $B_{P^{0}}$. Abusing notation, we let $\phi_{\lambda}=\phi_{\Lambda}$. In terms of the Fourier series $u(f)=\Sigma_{\Lambda} u_{\lambda} \phi_{\Lambda}\left(\Sigma_{\Lambda} f_{\lambda} \phi_{\Lambda}\right)$, (8) is equivalent to the ODE's

$$
\frac{d u_{\lambda}}{d t}-\lambda u_{\lambda}=f_{\lambda}, \frac{d^{2} u_{\lambda}}{d t^{2}}-\left(a_{1}-2 \beta\right) \frac{d u_{\lambda}}{d t}-\lambda u_{\lambda}=f_{\lambda} \text { for all } \lambda \in S p e c B_{P_{\beta}^{0}} \text { respectively. }
$$

Remark 2.5. Let $\sigma_{2}=I d$. In terms of the Fourier-coefficients, when $P^{0}$ is first-order,

$$
\begin{aligned}
|\xi|_{\widehat{W}_{0, \gamma, b-1}^{1,2, P_{\beta}^{0}}\left(C y l_{t_{0}}\right)}^{2}= & \Sigma_{\lambda \in \operatorname{Spec}\left(B_{P_{\beta}^{0}}\right), \lambda \neq 0}\left[\left(1+\lambda^{2}\right) \int_{t_{0}}^{\infty} \xi_{\lambda}^{2} s^{2 \gamma} d s+\int_{t_{0}}^{\infty}\left|\frac{d \xi_{\lambda}}{d s}\right|^{2} s^{2 \gamma} d s\right] \\
& +\Sigma_{\lambda \in \operatorname{Spec}\left(B_{P_{\beta}^{0}}\right), \lambda=0}\left[\int_{t_{0}}^{\infty} \xi_{\lambda}^{2} s^{2 b-2} d s+\int_{t_{0}}^{\infty}\left|\frac{d \xi_{\lambda}}{d s}\right|^{2} s^{2 b} d s\right] .
\end{aligned}
$$

When $P^{0}$ is second-order elliptic, using the usual $W^{2,2}$-elliptic estimate on strips, we routinely verify the following for any $\xi$ compactly supported in $C y l_{t_{0}+\epsilon}$.

$$
\begin{aligned}
|\xi|_{\widehat{W}_{0, \gamma, b-1}^{2,2, P_{\beta}^{0}}\left(C y l_{t_{0}}\right)}^{2} \leq & C(\epsilon)\left\{\Sigma_{\lambda \in \operatorname{Spec}\left(B_{P_{\beta}^{0}}\right), \lambda \neq 0} \int_{t_{0}}^{\infty}\left[\left(1+\lambda^{2}\right) \xi_{\lambda}^{2}+(1+|\lambda|)\left|\frac{d \xi_{\lambda}}{d t}\right|^{2}+\left|\frac{d^{2} \xi_{\lambda}}{d t^{2}}\right|^{2}\right] t^{2 \gamma} d t\right. \\
& \left.+\Sigma_{\lambda \in \operatorname{Spec}\left(B_{P_{\beta}^{0}}\right), \lambda=0}\left[\int_{t_{0}}^{\infty} \xi_{\lambda}^{2} t^{2 b-2} d s+\int_{t_{0}}^{\infty}\left(\left|\frac{d \xi_{\lambda}}{d t}\right|^{2}+\left|\frac{d^{2} \xi_{\lambda}}{d t^{2}}\right|^{2}\right) t^{2 b} d t\right]\right\}
\end{aligned}
$$

Remark 2.6. Multiplying by $e^{-\beta t}$ is a linear isomorphism:

$$
\widehat{C}_{-\beta, \gamma, b-1}^{k, \alpha, P^{0}} \longrightarrow \widehat{C}_{0, \gamma, b-1}^{k, \alpha, P_{\beta}^{0}}, \widehat{W}_{-\beta, \gamma, b-1}^{k, p, P^{0}} \longrightarrow \widehat{W}_{0, \gamma, b-1}^{k, p, P_{\beta}^{0}}, C_{-\beta, \gamma, b}^{k, \alpha} \longrightarrow C_{0, \gamma, b}^{k, \alpha}, L_{-\beta, \gamma, b}^{2} \longrightarrow L_{0, \gamma, b}^{2} .
$$

Definition 2.7. Suppose $\vec{\beta}=\left(\beta_{1}, \ldots ., \beta_{l_{0}}\right), \vec{\gamma}=\left(\gamma_{1}, \ldots \ldots, \gamma_{l_{0}}\right), \vec{b}=\left(b_{1}, \ldots \ldots, b_{l_{0}}\right)$ are vectors of $l_{0}$-entries. Given an ATID operator $P$ over a manifold $N$ with $l_{0}$ cylindrical ends, we denote the ends by $U_{j}, j=1 \ldots l_{0}$. We add the interior $U_{0}$ to obtain an open cover of $N$. Using a partition of unity $\chi_{j}, j=0 \ldots . . l_{0}$ subordinate to the cover, we define

$$
|\xi|_{\widehat{W}_{-\beta, \gamma, b-1}^{k, p, P}(N)}=\left|\chi_{0} \xi\right|_{W^{k, 2}\left(U_{0}\right)}+\sum_{j=1}^{l_{0}}\left|\chi_{j} \xi\right|_{\widehat{W}_{-\beta_{j}, \gamma_{j}, b_{j}-1}^{\left.k, p, U_{j}\right)}},
$$

where $P^{0, j}$ is the limit TID operator of $P$ on the $j$-th end. The same definition as (12) applies to all the other norms in Definition 2.3 (including $\widehat{C}_{-\beta, \gamma, b-1}^{k, \alpha, P}(N), C_{-\beta, \gamma, b}^{k, \alpha}(N)$ etc).

When the domain is the whole manifold, we usually hide the $N$ in the norm symbols.

Important Convention: When $\beta_{1}=\ldots=\beta_{l_{0}}=\beta$, we denote $\vec{\beta}$ (a vector) as $\beta$ (number). The same applies to $\vec{b}$ and $\vec{\gamma}$. This makes the notations consistent.

Definition 2.8. Let $\delta_{0}>0$ be small enough with respect to the data in Theorem 3.2 except $t_{0}$, such that the Neumann-Series in Lemma 4.4 and Theorem 3.3 converge as desired.

Let $k_{0} \geq 10,|\cdot|_{C^{k}}(t, y) \triangleq \Sigma_{0 \leq i+j \leq k}\left|\frac{\partial}{\partial t^{2}} \nabla^{j} \cdot\right|(t, y)$. We say that $P$ satisfies the $\left(\left._{\beta}\left(l_{1}, l_{2}\right)\right|_{C y l_{t_{0}}}\right.$-condition, if the following holds for any $k \leq k_{0}, t \geq t_{0}+1, \alpha \in[0,1), \xi$, and a $\delta_{0}$ small enough with respect to the data in Theorem 3.2

$$
\begin{gathered}
t^{l_{1}}\left|\left(P-P^{0}\right) \xi^{\perp_{\beta}}\right|_{C^{k, \alpha}\left(\bar{S}_{t}\right)} \leq \delta_{0}\left|\xi^{\perp_{\beta}}\right|_{C^{k+m_{0}, \alpha}\left(\bar{S}_{t}\right)}, t^{l_{1}}\left|\left(P-P^{0}\right) \xi^{\perp_{\beta}}\right|_{C^{k}}(t, y) \leq \delta_{0}\left|\xi^{\perp_{\beta}}\right|_{C^{k+m_{0}}}(t, y) \\
t^{l_{2}}\left|\left(P-P^{0}\right) \xi^{\|_{\beta}}\right|_{C^{k, \alpha}\left(\bar{S}_{t}\right)} \leq \delta_{0}\left|\xi^{\|_{\beta}}\right|_{C^{k+m_{0}, \alpha}\left(\bar{S}_{t}\right)}, t^{l_{2}}\left|\left(P-P^{0}\right) \xi^{\|_{\beta}}\right|_{C^{k}}(t, y) \leq \delta_{0}\left|\xi^{\|_{\beta}}\right|_{C^{k+m_{0}}}(t, y)
\end{gathered}
$$

We say that $P$ satisfies (S) $\left.(l)\right|_{C y l_{t_{0}}}$ if it satisfies $\left.S_{\beta}\left(l_{1}, l_{2}\right)\right|_{C y l_{t_{0}}}$ for all $\beta$ and $l_{1}=l_{2}=l$. We say that $P$ is $\vec{\beta}-A T I D$ on $N$ if for any $i$, it satisfies $\left.S_{\beta_{i}}(0,1)\right|_{C y l_{t_{0}}}$ for some $t_{0}$ on the $i$-th end. 
Remark 2.9. By our definition, $\delta_{0}$ depends on $P^{0}, B_{P^{0}}, \gamma, \beta, b$ etc.

Remark 2.10. It's easy to check (S $\left.(l)\right|_{C y l_{t_{0}}}$ for differential operators. In an arbitrary coordinate neighbourhood, write $P^{0}$ and $P$ as

$$
P^{0}=a_{0,1}(y) \frac{\partial}{\partial t}+\Sigma_{\gamma=2}^{n} a_{0, \gamma}(y) D^{\gamma} ; P=a_{1}(y, t) \frac{\partial}{\partial t}+\Sigma_{\gamma=2}^{n} a_{\gamma}(y, t) D^{\gamma} .
$$

Let $\delta_{1}$ be small enough with respect to the data in Theorem 3.2 (even smaller than $\delta_{0}$ ), then $P$ satisfies (S) $\left.(l)\right|_{C y l_{t_{0}}}$ if the following holds for all $y, k \leq k_{0}+1, t \geq t_{0}$.

$$
t^{l}\left|a_{\gamma}-a_{0, \gamma}\right|_{C^{k}}(t, y) \leq \delta_{1} \text { c.f. [11, (6.5)]. }
$$

A simple example of an $\left.S_{0}(0,1)\right|_{C y l_{t_{0}}}$-operator which does not satisfy (14) is $P^{0}+\delta_{0} \sin t \frac{\partial}{\partial t}$.

Defining $\left(\left.\square\right|_{-\vec{\beta}, \vec{\gamma}, \vec{b}} ^{\text {Sobolev, }}\right)$ and $\left(\square \mid \begin{array}{l}\text { Schauder } \\ -\vec{\beta}, \vec{\gamma}, \vec{b}\end{array}\right)$ as (2), Theorem 1.4 naturally generalizes to

Theorem 2.11. Let $m_{0}, k, k_{0}, \alpha, p$ be as in Theorem 1.4. Suppose $P$ is $\vec{\beta}-A T I D$ elliptic, and $\beta_{j}$ is not $P^{0, j}$-super indicial for any $j$. Then

- $\left(\left.\square\right|_{-\vec{\beta}, \vec{\gamma}, \vec{b}} ^{\text {Sobolev, }}\right)$ is Fredholm if for any $j, b_{j} \neq 1-\frac{1}{p}$ or $\beta_{j}$ is not $P^{0, j}-$ indicial;

- $\left(\square \mid \begin{array}{l}\text { Schauder } \\ -\vec{\beta}, \vec{\gamma}, \vec{b}\end{array}\right)$ is Fredholm if for any $j, b_{j} \neq 1$ or $\beta_{j}$ is not $P^{0, j}-$ indicial.

Dependence of the Constants: we follow the convention in [15, Definition 2.16,2.17]: the " $C$ " in a result (and the proof) depends on the data in the result, except the " $t_{0}$ " (initial time for the cylinders). We will add subscripts when $C$ depends on $t_{0}$ or other parameters. Remark 2.12. From now on, we hide the $P^{0}$ (or $P$ ) in the $\widehat{W}^{\prime} s\left(\widehat{C}^{\prime} s\right)$. The underlying operator should be clear from the context. When $\gamma=b$, we abbreviate $\widehat{W}_{-\beta, \gamma, b-1}^{k, p, P^{0}}, \widehat{C}_{-\beta, \gamma, b-1}^{k, \alpha, P^{0}}$, $W_{-\beta, \gamma, b}^{k, p, P^{0}}, C_{-\beta, \gamma, b}^{k, \alpha, P^{0}}$ to $\widehat{W}_{-\beta, b-1}^{k, p}, \widehat{C}_{-\beta, b-1}^{k, \alpha}, W_{-\beta, b}^{k, p}, C_{-\beta, b}^{k, \alpha}$ respectively. When $\gamma=b=0$, we further abbreviate $W_{-\beta, b}^{k, p}, C_{-\beta, b}^{k, \alpha}$ to $W_{-\beta}^{k, p}, C_{-\beta}^{k, \alpha}$.

\section{The Local inverses}

Definition 3.1. Let $\underline{\beta}<\beta$ be the indicial root adjacent to $\beta$ from below (but not equal to $\beta$ ), and $\bar{\beta}>\beta$ be the indicial root adjacent to $\beta$ from above.

Theorem 3.2. Let $P^{0}$ be a TID-operator, and $\beta$ be not $P^{0}$-super indicial. The following holds in view of Definition 1.1.

(i): When $b \neq \frac{1}{2}$ or $\beta$ is not $P^{0}$-indicial, $P^{0}: \widehat{W}_{-\beta, \gamma, b-1}^{m_{0}, 2}\left(C y l_{t_{0}}\right) \rightarrow L_{-\beta, \gamma, b}^{2}\left(C y l_{t_{0}}\right)$ admits a bounded linear right inverse. Let $Q_{\beta,+}^{P^{0}, t_{0}}\left(Q_{\beta,-}^{P^{0}, t_{0}}\right)$ denote the right inverse when $b>\frac{1}{2}\left(b<\frac{1}{2}\right)$ respectively when $\beta$ is indicial, and $Q_{\beta}^{P^{0}}, t_{0}$ denote the right inverse when $\beta$ is not indicial (When $\beta$ is not indicial, $Q_{\beta, \pm}^{P^{0}, t_{0}}$ both mean $Q_{\beta}^{P^{0}, t_{0}}$ ).

(ii): The following (regularity) estimates hold.

$$
\begin{aligned}
& \left|Q_{\beta,+}^{P^{0}, t_{0}} h\right|_{\widehat{C}_{-\beta, \gamma, b-1}^{k+m_{0}, \alpha}\left(\overline{C y l}_{t_{0}}\right)} \leq C|h|_{C_{-\beta, \gamma, b}^{k, \alpha}\left(\overline{C y l}_{t_{0}}\right)} \text { when } b>1 \text { and } \beta \text { is indicial; } \\
& \left|Q_{\beta,-}^{P^{0}, t_{0}} h\right|_{\widehat{C}_{-\beta, \gamma, 0}^{k+m_{0}, \alpha}\left(\overline{C y l}_{t_{0}}\right)} \leq C|h|_{C_{-\beta, \gamma, b}^{k, \alpha}\left(\overline{C y l}_{t_{0}}\right)} \text { when } b>1 \text { and } \beta \text { is indicial; } \\
& \left|Q_{\beta,-}^{P^{0}, t_{0}} h\right|_{\widehat{C}_{-\beta, \gamma, b-1}^{k+m_{0}, \alpha}\left(\overline{C y l}_{t_{0}}\right)} \leq C|h|_{C_{-\beta, \gamma, b}^{k, \alpha}\left(\overline{C y l}_{t_{0}}\right)} \text { when } b<1 \text { and } \beta \text { is indicial; } \\
& \left|Q_{\beta}^{P^{0}, t_{0}} h\right|_{C_{-\beta, \gamma}^{k+m_{0}, \alpha}\left(\overline{C y l}_{t_{0}}\right)} \leq C|h|_{C_{-\beta, \gamma}^{k, \alpha}\left(\overline{C y l}_{t_{0}}\right)} \text { when } \beta \text { is not indicial; } \\
& \left|Q_{\beta,+}^{P^{0}, t_{0}} h\right|_{\widehat{W}_{-\beta, \gamma, b-1}^{k+m_{0}, p}\left(\overline{C y l}_{t_{0}}\right)} \leq C|h|_{W_{-\beta, \gamma, b}^{k, p}\left(\overline{C y l}_{t_{0}}\right)} \text { when } b>1-\frac{1}{p} \text { and } \beta \text { is indicial; (19) } \\
& \left|Q_{\beta,-}^{P^{0}, t_{0}} h\right|_{\widehat{W}_{-\beta, \gamma, b-1}^{k+m_{0}, p}\left(\overline{C y l}_{t_{0}}\right)} \leq C|h|_{W_{-\beta, \gamma, b}^{k, p}\left(\overline{C y l}_{t_{0}}\right)} \text { when } b<1-\frac{1}{p} \text { and } \beta \text { is indicial; }(20) \\
& \left|Q_{\beta}^{P^{0}, t_{0}} h\right|_{W_{-\beta, \gamma}^{k+m_{0}, p}\left(\overline{C y l}_{t_{0}}\right)} \leq C|h|_{W_{-\beta, \gamma}^{k, p}\left(\overline{C y l}_{t_{0}}\right)} \text { when } \beta \text { is not indicial. }
\end{aligned}
$$


(iii): Suppose $\underline{\beta}$ is not super-indicial, then $Q_{\beta,+}^{P^{0}, t_{0}}=Q_{\underline{\beta},-}^{P^{0}, t_{0}}$ on $L_{-\underline{\beta}, b}^{2}\left(C y l_{t_{0}}\right)$ for any b.

Important Convention: through-out the article, we say that $h$ is in (or not in) a space if and only if the norm of $h$ is $<\infty(=\infty)$, respectively. Therefore all the estimates in Theorem 3.2 are regularity estimates.

Theorem 3.3. Suppose $P$ satisfies $\left.\mathrm{S}_{\beta}(0,1)\right|_{C y l_{t_{0}}}$ and $\beta$ is not $P^{0}$-super-indicial. Then except (16), $P$ also satisfies (i), (ii), (iii) in Theorem 3.2 (with $P^{0}$ replaced by $P$, and $Q_{\beta,+}^{P^{0}, t_{0}}, Q_{\beta,-}^{P^{0}, t_{0}}, Q_{\beta}^{P^{0}, t_{0}}$ replaced notationally by $\left.Q_{\beta,+}^{P, t_{0}}, Q_{\beta,-}^{P, t_{0}}, Q_{\beta}^{P, t_{0}}\right)$.

Remark 3.4. All the bounds in Theorem 3.2, 3.3 are independent of $t_{0}$.

Proof of Theorem 3.3 assuming [3.2: We momentarily hide $t_{0}, \beta, \pm$ in $Q_{\beta, \pm}^{P^{0}, t_{0}}$ in each case of Theorem 3.2. Theorem 3.2 and the $\beta-A T I D$ condition (Definition 2.8) implies when $\delta_{0}$ is sufficiently small, the Neumann-Series (c.f. [17, Theorem 2, page 69])

$$
\left[I d-Q^{P^{0}}\left(P^{0}-P\right)\right]^{-1} \triangleq \Sigma_{j=0}^{\infty}\left[Q^{P^{0}}\left(P^{0}-P\right)\right]^{j}
$$

converges to a two-sided inverse of $I d-Q^{P^{0}}\left(P^{0}-P\right)$. Hence $Q^{P} \triangleq\left(\sum_{j=0}^{\infty}\left[Q^{P^{0}}\left(P^{0}-P\right)\right]^{j}\right) Q^{P^{0}}$ is a right-inverse of $P$ i.e. $P Q^{P}=I d$, where we write $P=P^{0}\left[I d-Q^{P^{0}}\left(P^{0}-P\right)\right]$.

Lemma 3.5. (Hardy's inequality) For any $p \geq 2$,

$$
\begin{aligned}
& \int_{\frac{1}{10}}^{\infty}\left(t^{b-1} \int_{t}^{\infty}|f| d s\right)^{p} d t \leq C_{p, b} \int_{0}^{\infty}\left(t^{b}|f|\right)^{p} d t \text { when } b>1-\frac{1}{p} \\
& \int_{\frac{1}{10}}^{\infty}\left(t^{b-1} \int_{1}^{t}|f| d s\right)^{p} d t \leq C_{p, b} \int_{0}^{\infty}\left(t^{b}|f|\right)^{p} d t \text { when } b<1-\frac{1}{p}
\end{aligned}
$$

For all $b \in \mathbb{R}, p \geq 2, \vartheta \geq 0$, and $\mu \neq 0$, there exists a constant $C_{l_{\mu}, b}$ which depends only on $b$ and the lower bound on $|\mu|$ with the following properties.

$$
\begin{aligned}
\mu^{p(1+\vartheta)} \int_{\frac{1}{10}}^{\infty}\left(e^{\mu t} t^{b} \int_{t}^{\infty} e^{-\mu s}|f|(s-t)^{\vartheta} d s\right)^{p} d t & \leq C_{l_{\mu}, p, b} \int_{0}^{\infty}\left(|f| t^{b}\right)^{p} d t \text { when } \mu>0 ; \\
\mu^{p(1+\vartheta)} \int_{\frac{1}{10}}^{\infty}\left(e^{\mu t} t^{b} \int_{1}^{t} e^{-\mu s}|f|(t-s)^{\vartheta} d s\right)^{p} d t & \leq C_{l_{\mu}, p, b} \int_{0}^{\infty}\left(|f| t^{b}\right)^{p} d t \text { when } \mu<0
\end{aligned}
$$

(23) and (24) are special cases of [9, Theorem 330]. The proof for (25), (26) is elementary, we defer it to the Appendix.

The proof of [8, Lemma 6.37, Theorem 7.25] (reflection about the boundary) yields

Claim 3.6. Let $t_{0} \geq 2$. For any section $h \in C^{k, \alpha}\left(Y \times\left[t_{0}, t_{0}+1\right]\right)$ or $W^{k, p}\left[Y \times\left(t_{0}, t_{0}+1\right)\right]$, there exists an extension $h_{E, t_{0}}$ such that

- $h_{E, t_{0}}=0$ over $\left(0, t_{0}-0.01\right)$, and $h_{E, t_{0}}=h$ when $t \geq t_{0}$;

- $\left|h_{E, t_{0}}\right|_{C^{k, \alpha}\left[Y \times\left(0, t_{0}+1\right)\right]} \leq C|h|_{C^{k, \alpha}\left[Y \times\left(t_{0}, t_{0}+1\right)\right]},\left|h_{E, t_{0}}\right|_{W^{k, p}\left[Y \times\left(0, t_{0}+1\right)\right]} \leq C|h|_{W^{k, p}\left[Y \times\left(t_{0}, t_{0}+1\right)\right]}$;

- $h_{E, t_{0}}$ is translation-invariant in $t_{0}$ i.e. $h_{E, t_{0}}(f)(t)=h_{E, 2}\left(f_{t_{0}}\right)\left(t-t_{0}+2\right)$, where $f_{t_{0}}(t)=f\left(t+t_{0}-2\right)$.

We need to construct a linear operator $\dot{Q}_{\lambda}^{P_{\beta}^{0}}$ for each of the equations in (11), such that $u_{\lambda} \triangleq \dot{Q}_{\lambda}^{P_{\beta}^{0}} f_{\lambda}$ solves them respectively with required estimates. Summing the $\lambda$ 's up, we obtain the desired right inverse:

$$
\widetilde{Q}_{0, \pm}^{P_{\beta}^{0}} f \triangleq \Sigma_{\lambda \in \operatorname{Spec}\left(B_{P_{\beta}^{0}}\right)}\left(\dot{Q}_{\lambda}^{P_{\beta}^{0}} f_{\lambda}\right) \phi_{\lambda}
$$

When $\beta \neq 0$, it suffices to take

$$
\widehat{Q}_{\beta, \pm}^{P^{0}} \triangleq \sigma_{2}^{-1} \cdot e^{\beta t} \cdot \widetilde{Q}_{0, \pm}^{P_{\beta}^{0}} \cdot e^{-\beta t} \cdot \sigma_{1}^{-1} .
$$


Proof of Theorem 3.2 (i) for first-order operators: We construct the $\dot{Q}_{\lambda}^{P_{\beta}^{0}}$ as

\begin{tabular}{|l||l|l||c||l|l|}
\hline & & $u_{\lambda}\left(\triangleq \dot{Q}_{\lambda}^{P_{\beta}^{0}} f_{\lambda}\right)$ & & & $u_{\lambda} \triangleq \dot{Q}_{\lambda}^{P_{\beta}^{0}} f_{\lambda}$ \\
\hline 1 & $\lambda=0, b>\frac{1}{2}$ & $-\int_{t}^{\infty} f_{\lambda} d s$ & 3 & $\lambda>0$, all b & $-e^{\lambda t} \int_{t}^{\infty} e^{-\lambda s} f_{\lambda} d s$ \\
\hline 2 & $\lambda=0, b<\frac{1}{2}$ & $\int_{1}^{t} f_{\lambda} d s$ & 4 & $\lambda<0$, all b & $e^{\lambda t} \int_{1}^{t} e^{-\lambda s} f_{\lambda} d s$ \\
\hline
\end{tabular}

By Remark 2.5 and completeness of the spaces in Definition 2.3, it suffices to assume $f \in C_{c}^{\infty}\left(C y l_{1}\right)$, and only has finitely many non-zero Fourier coefficients. Without loss of generality, we only consider first-order operators, and assume that $\beta=0$ [see the derivation of (32)]. The proof for second-order operators is similar.

Applying the 4 inequalities in Lemma 3.5 to the 4 cases in (29) respectively, we find

$\int_{1}^{\infty} u_{\lambda}^{2} t^{2 b-2} d t \leq C \int_{1}^{\infty} f_{\lambda}^{2} t^{2 b} d t$ (in cases 1, 2), $\lambda^{2} \int_{1}^{\infty} u_{\lambda}^{2} t^{2 \gamma} d t \leq C \int_{1}^{\infty} f_{\lambda}^{2} t^{2 \gamma} d t$ (cases 3, 4).

Using the equation (11) to estimate $\frac{d u_{\lambda}}{d t}$, we trivially obtain

$\int_{1}^{\infty}\left|\frac{d u_{\lambda}}{d t}\right|^{2} t^{2 b} d t=\int_{1}^{\infty} f_{\lambda}^{2} t^{2 b} d t$ (in cases 1, 2), $\int_{1}^{\infty}\left|\frac{d u_{\lambda}}{d t}\right|^{2} t^{2 \gamma} d t=\int_{1}^{\infty} f_{\lambda}^{2} t^{2 \gamma} d t$ (cases 3, 4).

The above 4 estimates in the 4 cases yield

$$
\left|\widetilde{Q}_{0, b}^{P_{\beta}^{0}} f^{\perp_{0}}\right|_{W_{0, \gamma}^{m_{0}, 2}\left(C y l_{1}\right)} \leq C\left|f^{\perp_{0}}\right|_{L_{0, \gamma}^{2}\left(C y l_{1}\right)} ;\left|\widetilde{Q}_{0, b}^{P_{\beta}^{0}} f\right|_{\widehat{W}_{0, \gamma, b-1}^{m_{0}, 2}\left(C y l_{1}\right)} \leq C|f|_{L_{0, \gamma, b}^{2}\left(C y l_{1}\right)} .
$$

Using (28) $\left(\sigma_{i}^{-1}\right.$ are smooth) and the notation in Theorem $3.2 \mathbf{i}$, let $f=\sigma_{1}^{-1} h_{E, t_{0}} \in$ $C_{c}^{\infty}\left(C y l_{1}\right)$, we obtain

$$
\left|\widehat{Q}_{\beta, \pm}^{P^{0}} h_{E, t_{0}}\right|_{\widehat{W}_{-\beta, \gamma, b-1}^{m_{0}, 2}\left(C y l_{1}\right)} \leq C\left|h_{E, t_{0}}\right|_{L_{-\beta, \gamma, b}^{2}\left(C y l_{1}\right)} \text {. Let } Q_{\beta, \pm}^{P^{0}, t_{0}} h \triangleq \widehat{Q}_{\beta, \pm}^{P^{0}} h_{E, t_{0}},
$$

the following holds by Claim 3.6.

$$
\begin{aligned}
& \left|Q_{\beta, \pm}^{P^{0}, t_{0}} h\right|_{\widehat{W}_{-\beta, \gamma, b-1}^{m_{0}, 2}\left(C y l_{t_{0}}\right)} \leq\left|\widehat{Q}_{\beta, \pm}^{P^{0}, t_{0}} h_{E, t_{0}}\right|_{\widehat{W}_{-\beta, \gamma, b-1}^{m_{0}, 2}\left(C y l_{2}\right)} \leq C\left|h_{E, t_{0}}\right|_{L_{-\beta, \gamma, b}^{2}\left(C y l_{1}\right)} \\
\leq & C|h|_{L_{-\beta, \gamma, b}^{2}\left(C y l_{t_{0}}\right)} .
\end{aligned}
$$

The above means $Q_{\beta, \pm}^{P^{0}, t_{0}}$ is bounded.

Similar ideas apply to second-order equations, we defer the detail to the Appendix. By our constructions in (29), (64), (67), (28), we routinely verify Theorem 3.2 (iii).

Proof of Theorem 3.2 (18), (21): It suffices to apply Maz'ya-Plamenevskii's trick ([13, Lemma 1.1, 4.1]). We adopt (30) and assume $k=0$. Theorem 3.2 (i) yields

$$
\begin{aligned}
& \int_{l-1}^{l+1}\left|\widetilde{Q}_{0}^{P_{\beta}^{0}}\left(\xi_{m} f\right)\right|_{L^{2}(Y)}^{2} t^{2 \gamma} d t \leq C e^{2 \mu_{0} l} \int_{l-1}^{l+1}\left|\widetilde{Q}_{0}^{P_{\beta}^{0}}\left(\xi_{m} f\right)\right|_{L^{2}(Y)}^{2} e^{-2 \mu_{0} t} t^{2 \gamma} d t \\
\leq & C e^{2 \mu_{0} l} \int_{1}^{\infty}\left|\xi_{m} f\right|_{L^{2}(Y)}^{2} e^{-2 \mu_{0} t} t^{2 \gamma} d t \leq C e^{2 \mu_{0}(l-m)} \int_{m-2}^{m+2}|f|_{L^{2}(Y)}^{2} t^{2 \gamma} d t \\
\leq & C e^{-2\left|\mu_{0}\right||l-m|} \sup _{m}\left|t^{\gamma} f\right|_{L^{2}\left(2 S_{m}\right)}^{2}, \\
& \left|\widetilde{Q}_{0}^{P_{\beta}^{0}} f\right|_{L_{0, \gamma}^{2}\left(C y l_{1}\right)} \leq C|f|_{L_{0, \gamma}^{2}\left(C y l_{1}\right)},
\end{aligned}
$$


where we let $\left|\mu_{0}\right|$ be small enough with respect the spectrum gap, and the sign of $-\mu_{0}$ be the same as that of $l-m$ (when $l=m$ either sign works). Summing the $m$ in (34) over all integers $\geq 3$, we obtain

$$
\left|\widetilde{Q}_{0}^{P_{\beta}^{0}} f\right|_{L_{0, \gamma}^{2}\left(S_{l}\right)} \leq C \sup _{m}\left|t^{\gamma} f\right|_{L^{2}\left(2 S_{m}\right)} \Sigma_{m \geq 2} e^{-\left|\mu_{0}\right||l-m|} \leq C|f|_{C_{0, \gamma}^{0}\left(C y l_{1}\right)} \text { for any } l \geq 2 .
$$

We recall $\left|\widetilde{Q}_{0}^{P_{\beta}^{0}} f\right|_{L^{2}\left(S_{l}\right)} \leq C l^{-\gamma}\left|\widetilde{Q}_{0}^{P_{\beta}^{0}} f\right|_{L_{0, \gamma}^{2}\left(S_{l}\right)},|f|_{C^{\alpha}\left(S_{l}\right)} \leq C l^{-\gamma}|f|_{C_{0, \gamma}^{\alpha}\left(S_{l}\right)}$, $|f|_{L^{p}\left(S_{l}\right)} \leq C l^{-\gamma}|f|_{L_{0, \gamma}^{p}\left(S_{l}\right)}$, and the following standard (Schauder and $L^{p}$ ) estimate on $S_{l}$

$$
|\xi|_{C^{1, \alpha}\left(\frac{S_{l}}{2}\right)} \leq C\left|P_{\beta}^{0} \xi\right|_{C^{\alpha}\left(S_{l}\right)}+C|\xi|_{L^{2}\left(S_{l}\right)},|\xi|_{W^{1, p}\left(\frac{S_{l}}{2}\right)} \leq C\left|P_{\beta}^{0} \xi\right|_{L^{p}\left(S_{l}\right)}+C|\xi|_{L^{2}\left(S_{l}\right)} .
$$

Then we obtain from (34) and (35) that

$$
\begin{array}{r}
l^{\gamma}\left|\widetilde{Q}_{0}^{P_{\beta}^{0}} f\right|_{C^{1, \alpha}\left(\frac{S_{l}}{2}\right)} \leq C\left[|f|_{C_{0, \gamma}^{\alpha}\left(\overline{C y l}_{1}\right)}+|f|_{C_{0, \gamma}^{0}\left(S_{l}\right)}\right] \leq C|f|_{C_{0, \gamma}^{\alpha}\left(\overline{C y l_{1}}\right)}, \\
l^{\gamma}\left|\widetilde{Q}_{0}^{P_{\beta}^{0}} f\right|_{W^{1, p}\left(\frac{S_{l}}{2}\right)} \leq C\left[|f|_{L_{0, \gamma}^{p}\left(S_{l}\right)}+\left|\widetilde{Q}_{0}^{P_{\beta}^{0}} f\right|_{L_{0, \gamma}^{2}\left(S_{l}\right)}\right] .
\end{array}
$$

Taking $\sup _{l \geq 2}$ of (38) and $\Sigma_{l \geq 2}$ of (39), we obtain by Definition 2.3 and (35) that

$$
\left|\widetilde{Q}_{0}^{P_{\beta}^{0}} f\right|_{C_{0, \gamma}^{1, \alpha}\left(\overline{C y l_{2}}\right)} \leq C|f|_{C_{0, \gamma}^{\alpha}\left(\overline{C y l_{1}}\right)},\left|\widetilde{Q}_{0}^{P_{\beta}^{0}} f\right|_{W_{0, \gamma}^{1, p}\left(C y l_{2}\right)} \leq C|f|_{L_{0, \gamma}^{p}\left(C y l_{1}\right)} .
$$

By the same argument in (33) [using (40) instead of [32)], we obtain (18) and (21).

Proof of Theorem 3.2 (15), (16), (17), (19), (20): We adopt (30). Using Lemma 3.5 and (29), we find the simple estimates

$$
\begin{aligned}
& \left|Q_{+}^{P_{\beta}^{0}} f^{\|_{0}}\right| \leq C\left|\int_{t}^{\infty} f^{\|_{0}} d s\right| \leq C\left|f^{\|_{0}}\right|_{C_{0, b}^{0}\left(\overline{C y l_{t}}\right)}\left|\int_{t}^{\infty} s^{-b} d s\right| \leq C\left|f^{\|_{0}}\right|_{C_{0, b}^{0}\left(\overline{C y l_{t}}\right)} t^{1-b} \text { when } b>1, \\
& \left|Q_{-}^{P_{\beta}^{0}} f^{\|_{0}}\right| \leq C\left|\int_{1}^{t} f^{\|_{0}} d s\right| \leq C\left|f^{\|_{0}}\right|_{C_{0, b}^{0}\left(\overline{C y l_{1}}\right)}\left|\int_{1}^{t} s^{-b} d s\right| \leq\left\{\begin{array}{cc}
C\left|f^{\|_{0}}\right|_{C_{0, b}^{0}}\left(\overline{C y l_{1}}\right)^{1-b} & \text { when } b<1, \\
C\left|f^{\|_{0}}\right|_{C_{0, b}^{0}}^{0}\left(\overline{C y l_{1}}\right) & \text { when } b>1 .
\end{array}\right. \\
& \left(\int_{1}^{\infty}\left(t^{b-1}\left|Q_{ \pm}^{P_{\beta}^{0}} f^{\|_{0}}\right|\right)^{p} d t\right)^{\frac{1}{p}} \leq C\left(\int_{1}^{\infty}\left(t^{\gamma}\left|f^{\|_{0}}\right|\right)^{p} d t\right)^{\frac{1}{p}} \text { when } b>(<) 1-\frac{1}{p} \text { respectively. }
\end{aligned}
$$

Combining $\frac{\partial}{\partial t} Q_{ \pm}^{P_{\beta}^{0}} f^{\|_{0}}=f^{\|_{0}}$, we find

$$
\left\{\begin{array}{c}
\left|Q_{+}^{P_{\beta}^{0}} f^{\|_{0}}\right|_{\widehat{C}_{0, b-1}^{1, \alpha}\left(\overline{C y l_{2}}\right)} \leq C\left|f^{\|_{0}}\right|_{C_{0, b}^{\alpha}\left(\overline{C y l_{1}}\right)} \text { when } b>1(b<1) \text { respectively, } \\
\left|Q_{ \pm}^{P_{\beta}^{0}} f^{\|_{0}}\right|_{\widehat{W}_{0, b-1}^{1, p}\left(C y l_{2}\right)} \leq C\left|f^{\|_{0}}\right|_{L_{0, b}^{p}\left(C y l_{1}\right)} \text { when } b>1-\frac{1}{p}\left(b<1-\frac{1}{p}\right) \text { respectively, } \\
\left|Q_{-}^{P_{\beta}^{0}} f^{\|_{0}}\right|_{\widehat{C}_{0,0}^{1, \alpha}\left(\overline{C y l}_{2}\right)} \leq C\left|f^{\|_{0}}\right|_{C_{0, b}^{\alpha}\left(\overline{C y l}_{1}\right)} \text { when } b>1 .
\end{array}\right.
$$

Because $Q_{ \pm}^{P_{\beta}^{0}} f^{\perp_{0}}$ is perpendicular to the kernel, (31) and the proof of (18), (21) yield

$$
\left|\widetilde{Q}_{0, \pm}^{P_{\beta}^{0}} f^{\perp_{0}}\right|_{C_{0, \gamma}^{1, \alpha}\left(\overline{C y l_{2}}\right)} \leq C\left|f^{\perp_{0}}\right|_{C_{0, \gamma}^{\alpha}\left(\overline{C y l_{1}}\right)},\left|\widetilde{Q}_{0, \pm}^{P_{\beta}^{0}} f^{\perp_{0}}\right|_{W_{0, \gamma}^{1, p}\left(C y l_{2}\right)} \leq C\left|f^{\perp_{0}}\right|_{L_{0, \gamma}^{p}\left(C y l_{1}\right)} .
$$

(42), (43) amount to (the special cases of) (15), (16), (17), (19), (20) with $P^{0}$ replaced by $P_{\beta}^{0}, \beta$ by 0 . The argument in (32), (33) yields the desired five estimates in general.

\section{Regularity and proof of Theorem 1.4, 2.11}

Remark 4.1. Without loss of generality, in the proof of Claim 4.2, Lemma 4.4, and Proposition 4.5. we only consider first-order operators, and assume $k=t_{0}=1, \gamma=b$ (see Remark 2.12). The proof for the other cases is absolutely the same. Though second-order elliptic operators are more complicated [there are 2 homogeneous solutions to the second-order ODEs in (11)], the desired regularity still follows in the same way. 
Claim 4.2. Given any TID-operator $P^{0}$, suppose $\beta, \beta$ are not $P^{0}$-super indicial. Then for any $t_{0}, k \geq 1, \epsilon>0$, the following estimate holds uniformly in $\left.h \in \operatorname{kerP}^{0}\right|_{L_{-\beta, \gamma,-\frac{1}{2}+\epsilon}^{2}}\left(C y l_{t_{0}}\right)$ :

$$
|h|_{C_{-\underline{\beta}}^{k, \alpha}\left(\overline{\left.C y l_{t_{0}+\epsilon}\right)}\right.} \leq C_{t_{0}}|h|_{L_{-\beta, \gamma,-\frac{1}{2}+\epsilon}^{2}}\left(C y l_{t_{0}}\right) .
$$

Proof: We adopt Remark 4.1. The condition $|h|_{L_{-\beta,-\frac{1}{2}+\epsilon}^{2}}\left(C y l_{1}\right)<\infty\left(h \in \operatorname{KerP}^{0}\right)$ implies

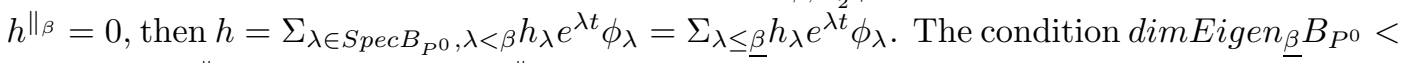
$\infty$ implies $\left|h^{\|\|_{\beta}}\right|_{C_{-\underline{\beta}}^{1, \alpha}\left(\overline{C y l_{1+\epsilon}}\right)} \leq C\left|h^{\|_{\beta}}\right|_{L_{-\beta,-\frac{1}{2}+\epsilon}^{2}}\left(C y l_{1}\right)$.

Since $h^{\perp_{\beta}} \in \operatorname{Ker} \widehat{B}_{P_{\beta}^{0}}$, using the Schauder estimate in (37) like (39), the rate of decay is improved i.e. $\left|h^{\perp_{\beta}}\right|_{C_{-\underline{\beta}}^{1, \alpha}\left(\overline{C y l}_{1+\epsilon}\right)} \leq C\left|h^{\perp_{\beta}}\right|_{L_{-\underline{\beta}}^{2}\left(C y l_{1+\frac{\epsilon}{2}}\right)} \leq C\left|h^{\perp_{\beta}}\right|_{L_{-\beta,-\frac{1}{2}+\epsilon}^{2}}\left(C y l_{1}\right)$. Thus

$$
\begin{aligned}
& \left.|h|_{C_{-\underline{\beta}}^{1, \alpha}\left(\overline{C y l}_{1+\epsilon}\right)} \leq\left|h^{\perp_{\beta}}\right|_{C_{-\underline{\beta}}^{1, \alpha}\left(\overline{C y l}_{1+\epsilon}\right)}+\left|h^{\|\|_{\beta}}\right|_{C_{-\underline{\beta}}^{1, \alpha}\left(\overline{C y l_{1+\epsilon}}\right)}\right) \\
\leq & C\left(\left|h^{\perp_{\beta}}\right|_{L_{-\beta,-\frac{1}{2}+\epsilon}^{2}}\left(C y l_{1}\right)+\left|h^{\|_{\beta}}\right|_{L_{-\beta,-\frac{1}{2}+\epsilon}^{2}}\left(C y l_{1}\right)\right) \\
\leq & C|h|_{L_{-\beta,-\frac{1}{2}+\epsilon}^{2}}\left(C y l_{1}\right) .
\end{aligned}
$$

Definition 4.3. $S_{\beta, \pm}^{P^{0}, t_{0}} \triangleq Q_{\beta, \pm}^{P^{0}, t_{0}} P^{0}-I d$ is bounded from $\widehat{W}_{-\beta, \gamma,-\frac{1}{2} \pm \epsilon}^{m_{0}, 2}\left(C y l_{t_{0}}\right)$ to itself. We verify $S_{\beta, \pm}^{P, t_{0}} \triangleq Q_{\beta, \pm}^{P, t_{0}} P-I d=\left(\Sigma_{j=0}^{\infty}\left[Q_{\beta, \pm}^{P^{0}, t_{0}}\left(P^{0}-P\right)\right]^{j}\right) S_{\beta, \pm}^{P^{0}, t_{0}}$.

Lemma 4.4. Let $P$ be an operator on $C y l_{t_{0}}$ as in Definition $[2.8$. Suppose $\beta, \underline{\beta}$ are not $P^{0}$-super indicial, then the following hold for any $\epsilon>0, t_{0} \geq 2, \gamma$, and $k \leq k_{0}+\bar{m}_{0}-1$.

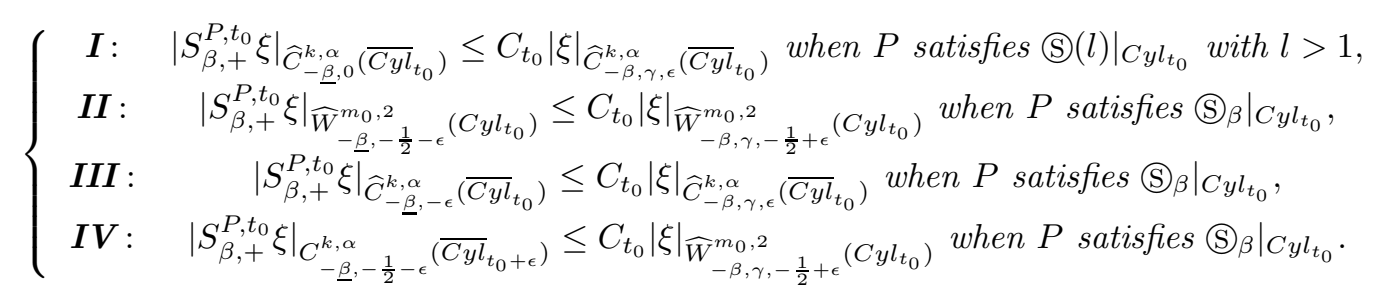

Proof. We adopt Remark 4.1 and only prove $\mathbf{I}$ and IV. II and III are similar (to I). First, we deal with the model operator $S_{\beta,+}^{P^{0}, t_{0}}$. Because $S_{\beta,+}^{P^{0}, 2} \xi \in \operatorname{Ker} P^{0}$, Claim 4.2 yields

$$
\begin{aligned}
& \left|S_{\beta,+}^{P^{0}, 2} \xi\right|_{\widehat{C}_{-\beta, 0}^{1, \alpha}\left(\overline{C y l_{2}}\right)} \leq C\left|S_{\beta,+}^{P^{0}, 2} \xi\right|_{\widehat{C}_{-\beta, \epsilon}^{1, \alpha}(Y \times[2,4])}+\left|S_{\beta,+}^{P^{0}, 2} \xi\right|_{\widehat{C}_{-\beta, 0}^{1, \alpha}\left(\overline{C y l_{3}}\right)} \\
\leq & C\left|S_{\beta,+}^{P^{0}, 2} \xi\right|_{\widehat{C}_{-\beta, \epsilon}^{1, \alpha}\left(\overline{C y l_{2}}\right)} \leq C\left|Q_{\beta,+}^{P^{0}, 2} P^{0} \xi\right|_{\widehat{C}_{-\beta, \epsilon}^{1, \alpha}\left(\overline{C y l_{2}}\right)}+C|\xi|_{\widehat{C}_{-\beta, \epsilon}^{1, \alpha}\left(\overline{C y l_{2}}\right)} \\
\leq & C|\xi|_{\widehat{C}_{-\beta, \epsilon}^{1, \alpha}\left(\overline{C y l_{2}}\right)} .
\end{aligned}
$$

When $P$ satisfies (S) $\left.(l)\right|_{C y l_{1}}$ with $l>1,\left|\left(P^{0}-P\right) \eta\right|_{C_{-\underline{\beta}, l}^{\alpha}\left(\overline{C y l_{2}}\right)} \leq C \delta_{0}|\eta|_{C_{-\underline{\beta}}^{1, \alpha}\left(\overline{\left.C y l_{2}\right)}\right.}$ for any $\eta$. Hence we obtain the following (by Theorem 3.2 (16) and the above).

$$
\left|Q_{\beta,+}^{P^{0}, 2}\left(P^{0}-P\right) \eta\right|_{\widehat{C}_{-\underline{\beta}, 0}^{1, \alpha}\left(\overline{C y l}_{2}\right)}=\left|Q_{\underline{\beta},-}^{P^{0}, 2}\left(P^{0}-P\right) \eta\right|_{\widehat{C}_{-\underline{-\beta}, 0}^{1, \alpha}\left(\overline{C y l_{2}}\right)} \leq C \delta_{0}|\eta|_{C_{-\underline{\beta}}^{1, \alpha}\left(\overline{C y l}_{2}\right)} .
$$

Let $\delta_{0}$ be small enough such that $C \delta_{0} \leq \frac{1}{2}$ in the above, the Neumann series converges i.e.

$$
\left|\left(\Sigma_{j=0}^{\infty}\left[Q_{\beta,+}^{P^{0}, 2}\left(P^{0}-P\right)\right]^{j}\right) \eta\right|_{\widehat{C}_{-\underline{-\alpha}, 0}^{1, \alpha}\left(\overline{C y l}_{2}\right)} \leq C|\eta|_{C_{-\underline{\alpha}}^{1, \alpha}\left(\overline{C y l_{2}}\right)} .
$$

Let $\eta=S_{\beta,+}^{P^{0}, 2} \xi$ in (46), Lemma 4.4 $\mathbf{I}$ follows from (44) and Definition 4.3.

On IV, using the Schauder estimate (37) and II (note $S_{\beta,+}^{P, 2} \xi \in \operatorname{KerP}$ ), we obtain

$$
\left|S_{\beta,+}^{P, 2} \xi\right|_{C^{k, \alpha}\left(\frac{\epsilon S_{l}}{2}\right)} \leq C\left|S_{\beta,+}^{P, 2} \xi\right|_{L^{2}\left(\epsilon S_{l}\right)} \leq C l^{\frac{1}{2}+\epsilon} e^{\underline{\beta}}\left|S_{\beta,+}^{P, 2} \xi\right|_{L_{-\underline{\beta},-\frac{1}{2}-\epsilon}^{2}\left(\epsilon S_{l}\right)} .
$$

By Definition 2.7, the proof of $\mathbf{I V}$ is complete. 
Proposition 4.5. Under the same conditions on $P, \epsilon, k, \gamma, \beta, \underline{\beta}$ in Lemma 4.4, the following hold uniformly for any $\left.h \in \operatorname{Ker} P\right|_{\widehat{W}_{-\beta, \gamma,-\frac{1}{2}+\epsilon}^{m_{0}, 2}}\left(C y l_{t_{0}}\right)$.

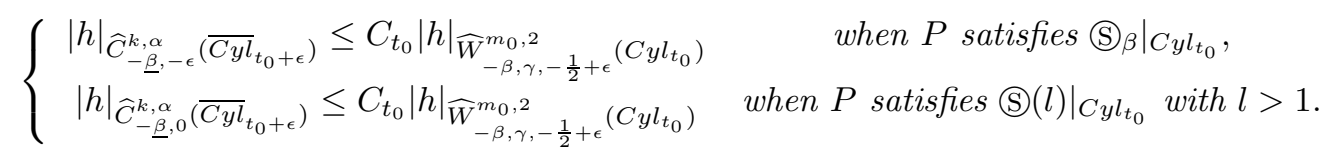

Consequently, for any $k \leq k_{0}-2$, suppose the signs of $\mu_{1}$ and $\mu_{2}$ are the same, we have

$$
(\text { ker } \mid \text { Coker } \mid \text { Index })\left(\left.\square\right|_{-\beta, \gamma, 1-\frac{1}{p}+\mu_{1}} ^{\text {Sobolev } p}\right)=(\text { ker } \mid \text { Coker } \mid \text { Index })\left(\left.\square\right|_{-\beta, \gamma, 1+\mu_{2}} ^{\text {Schauder }}\right) \text { respectively. }
$$

Moreover, suppose $\underline{\beta}<\bar{\beta}$ are 2 adjacent indicial roots which are not $P^{0}$-super indicial, then

$$
\begin{aligned}
& (\text { ker } \mid \text { Coker } \mid \text { Index })\left(\left.\square\right|_{-\bar{\beta}, \gamma_{1}, 1-\frac{1}{p}+\epsilon_{1}} ^{\text {Sobolev,p }}\right)=(\text { ker } \mid \text { Coker } \mid \text { Index })\left(\left.\square\right|_{-\beta, \gamma_{2}, b} ^{\text {Sobolev }, p}\right) \\
& =(\text { ker } \mid \text { Coker } \mid \text { Index })\left(\left.\square\right|_{-\underline{\beta}, \gamma_{3}, 1-\frac{1}{p}-\epsilon_{2}} ^{\text {Sobolp }, p}\right) \text {. } \\
& (\text { ker } \mid \text { Coker } \mid \text { Index })\left(\left.\square\right|_{-\bar{\beta}, \gamma_{1}, 1+\epsilon_{1}} ^{\text {Schauder }}\right)=(\text { ker } \mid \text { Coker } \mid \text { Index })\left(\left.\square\right|_{-\beta, \gamma_{2}, b} ^{\text {Schauder }}\right) \\
& =(\text { ker } \mid \text { Coker } \mid \text { Index })\left(\left.\square\right|_{-\underline{\beta}, \gamma_{3}, 1-\epsilon_{2}} ^{\text {Schauder }}\right) \text {. }
\end{aligned}
$$

for any $p \geq 2, b, \gamma_{i}(i=1,2,3), \beta \in(\underline{\beta}, \bar{\beta})$, and $\epsilon_{1}, \epsilon_{2}>0$.

Proof: It's a direct corollary of Lemma 4.4 We only prove the second assertion, the first is easier. We adopt Remark 4.1. We note that $h \in K \operatorname{KerP}$ implies

$$
h=-S_{\beta,+}^{P, t} h \text { for all } t \geq t_{0} .
$$

Let $t=t_{0}$, Lemma 4.4 (IV) says $|h|_{\widehat{C}_{-\underline{-},-2}^{k, \alpha}\left(\overline{C y l}_{t_{0}+\epsilon}\right)} \leq C|h|_{\widehat{W}_{-\beta,-\frac{1}{2}+\epsilon}^{m_{0}, 2}}\left(C y l_{t_{0}}\right)$. Let $t=t_{0}+\epsilon$ in (49), Lemma 4.4 (I) and the above imply

$$
|h|_{\widehat{C}_{-\underline{\beta}, 0}^{k, \alpha}\left(\overline{C y l}_{t_{0}+\epsilon}\right)} \leq C|h|_{\widehat{C}_{-\underline{\beta},-2}^{k, \alpha}\left(\overline{C y l_{t_{0}+\epsilon}}\right.} \leq C|h|_{\widehat{W}_{-\beta,-\frac{1}{2}+\epsilon}^{m_{0}, 2}\left(C y l_{t_{0}}\right.} .
$$

Proof of Theorem 1.4, 2.11]: With the help of Lemma 4.4, it is standard. We only do the argument for 1.4, and only show the $\widehat{W}(\widehat{C})$ theory for $b>1-\frac{1}{p}(b>1)$, respectively.

By [11, (2.5) to line 2, page 421] (also see [15, Theorem 4.14]), piecing together the local right inverses in Theorem 3.3 on the ends, we obtain a global parametrix $Q_{\beta,+, N}$ such that

$$
\left\{\begin{array}{l}
Q_{\beta,+, N} P=I d+S_{\text {left }}, \\
P Q_{\beta,+, N}=I d+S_{\text {right }}
\end{array}, S_{\text {right }} \text { is compact from } W_{-\beta, \gamma, b}^{k, p}\left(C_{-\beta, \gamma, b}^{k, \alpha}\right)\right. \text { to itself. }
$$

Lemma 4.4 IV says $S_{\text {left }}$ is bounded from $\widehat{W}_{-\beta, \gamma, b-1}^{m_{0}, 2}$ to $\widehat{C}_{-\underline{\beta},-1}^{k_{0}+m_{0}-1, \alpha}$. Since the weight is improved, by the (cylindrical analogue of) [15, proof of Lemma 4.10], $\widehat{C}_{-\underline{\beta},-1}^{k_{0}+m_{0}-1, \alpha}$ embeds compactly into $\widehat{W}_{-\beta, \gamma, b-1}^{k+m_{0}, p}$ and $\widehat{C}_{-\beta, \gamma, b-1}^{k+m_{0}, \alpha}$ when $k \leq k_{0}-2$. Then $S_{\text {left }}$ is compact from $\widehat{W}_{-\beta, \gamma, b-1}^{k+m_{0}, p}\left(\widehat{C}_{-\beta, \gamma, b-1}^{k+m_{0}, \alpha}\right)$ to itself. Hence [18, Theorem 4.6.5] implies $P$ is Fredholm.

\section{Index}

Definition 5.1. Let $B$ be an operator as in Proposition [5.4. Let $d_{\lambda} \triangleq \operatorname{dimker}(B-\lambda I d)$. Let $\operatorname{Eta}(B)$ denote the eta-invariant defined in [3, Theorem 3.10 (iii)], and

$$
H_{\beta, B} \triangleq\left\{\begin{array}{cc}
-\frac{d_{0}}{2}-\Sigma_{0>\lambda \geq \beta} d_{\lambda} & \text { when } \beta<0, \\
-\frac{d_{0}}{2} & \text { when } \beta=0, \\
\frac{d_{0}}{2}+\Sigma_{0<\lambda<\beta} d_{\lambda} & \text { when } \beta>0 .
\end{array}\right\}, H_{\beta, B, b} \triangleq\left\{\begin{array}{cc}
H_{\beta, B} & \text { when } b>\frac{1}{2}, \\
H_{\beta, B}+d_{\beta} & \text { when } b<\frac{1}{2} .
\end{array}\right.
$$


Theorem 5.2. Under the conditions in Theorem 2.11, suppose in addition that $P$ is firstorder elliptic, $\sigma_{1}$ in (1) is an isometry, and $P$ is translation invariant on each end. Then

$$
\text { index }\left.P\right|_{\widehat{W}_{-\vec{\beta}, \vec{b}-1}^{1,2} \rightarrow L_{-\vec{\beta}, \vec{b}}^{2}}=\int_{X} \alpha_{0} d v o l-\frac{\operatorname{Eta}\left(B_{P^{0}}\right)}{2}+\sum_{j=1}^{l_{0}} H_{\beta_{j}, B_{P^{0}, j}^{j}, b_{j}} \text {, where }
$$

$\alpha_{0}$ is the $0-$ th order term in the expansion of the kernel of $e^{-t \widetilde{P}^{\star} \widetilde{P}}-e^{-t \widetilde{P} \widetilde{P}^{\star}}, \widetilde{P}$ is the double of $P$ on the double of $N_{0}\left(N_{0} \sharp \bar{N}_{0}\right)$.

Remark 5.3. When $P$ satisfies proper conditions as Definition 2.8 under the operator norm, we can usually deform it continuously to be translation-invariant on each end. Thus the index of $P$ can still be computed by deformation invariance.

Proof of Theorem 5.2: Without loss of generality, we assume $\sigma_{2}=I d$. We recall the "Extended $L^{2}$-sections" defined in [3, first paragraph of page 58], and note that the dual of $L_{0, b}^{2}$ is isomorphic to $L_{0,-b}^{2}$. Using Claim 4.2 for both $L$ and $L^{\star}=-\left(\frac{\partial}{\partial t}+B\right) \sigma_{1}^{-1}$, we have

$$
\begin{gathered}
\left.\operatorname{Ker} L\right|_{\widehat{W}_{0, b-1}^{1,2}}=\left.\operatorname{Ker} L\right|_{L^{2}(N, E)},\left.\operatorname{Ker} L^{\star}\right|_{L_{0,-b}^{2}}=\left.K \operatorname{Ker} L^{\star}\right|_{\text {Extended } L^{2}(N, F)} \text { when } b>\frac{1}{2} ; \\
\left.\operatorname{Ker} L\right|_{\widehat{W}_{0, b-1}^{1,2}}=\left.\operatorname{Ker} L\right|_{\text {Extended } L^{2}(N, E)},\left.\operatorname{Ker} L^{\star}\right|_{L_{0,-b}^{2}}=\left.\operatorname{Ker} L^{\star}\right|_{L^{2}(N, F)} \text { when } b<\frac{1}{2} . \\
\left.\operatorname{Index} L\right|_{\widehat{W}_{0, b-1}^{1,2} \rightarrow L_{0, b}^{2}}=\left\{\begin{array}{l}
h(E)-h(F)-h_{\infty}(F) \text { when } b>\frac{1}{2} ; \\
h(E)-h(F)+h_{\infty}(E) \text { when } b<\frac{1}{2},
\end{array}\right.
\end{gathered}
$$

where $h(E), h(F), h_{\infty}(F), h_{\infty}(F)$ are defined in [3, Corollary (3.14)]. Assuming $\vec{\beta}=0$ for all $j$, (51) follows from [3, Corollary (3.14), (3.25)].

By Proposition 4.5 (c.f. Remark 1.7),

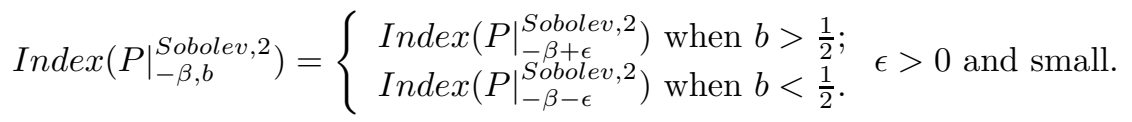

The index change formula of Lockhart-McOwen [11, Theorem 8.1] means for any $i_{0}$, let $\beta_{i}, i \neq i_{0}$ be unaltered, and $\beta_{i_{0}}$ go across an eigen-value $\lambda$ of $B_{i_{0}}$ (from $\lambda+\epsilon$ to $\lambda-\epsilon$ ), the index decreases by $\operatorname{dimker}\left(B_{P^{0}}^{i_{0}}-\lambda I d\right)$. The proof for general $\vec{\beta}$ is complete with the help of (54) [and (51) for $\vec{\beta}=0$ ].

Proposition 5.4. In the setting of Definition 1.1, suppose $B$ is a self-adjoint first-order elliptic differential operator on $E \rightarrow Y$. Then $\operatorname{Eta}(B-\beta I d)=E t a(B)-2 H_{\beta, B}-d_{\beta}$.

Proof. We form the full cylinder $Y \times(-\infty,+\infty)_{t}$ and consider $P=\frac{\partial}{\partial t}-B$. We consider the open cover $E n d_{+}, Y \times(-4,4), E n d_{-}$, where $E n d_{+}=C y l_{3}$ and $E n d_{-}=Y \times(-\infty,-3)$. In $E n d_{-}$, under the coordinate $s=-t, P=-\frac{\partial}{\partial s}-B=-\left[\frac{\partial}{\partial s}-(-B)\right]$. Let $-\vec{\beta}=(-\beta, 0)$ $\left(e^{-\beta t}\right.$ on $E n d_{+}$and 1 on $\left.E n d_{-}\right)$, Theorem 5.2 says when $b>\frac{1}{2}$ that

$$
\left.\operatorname{index} P\right|_{\widehat{W}_{-\vec{\beta}, b-1}^{1,2} \rightarrow L_{-\vec{\beta}, b}^{2}}=\int_{X} \alpha_{0} \operatorname{dvol}-\frac{\operatorname{Eta}(B)}{2}-\frac{\operatorname{Eta}(-B)}{2}-\frac{d_{0}}{2}+H_{\beta, B} .
$$

On the other hand, let $\rho$ be a smooth function which is equal to $e^{\beta t}$ on $E n d_{+}$, and 1 on End $d_{-}$, the conjugation $P_{\rho}=\frac{1}{\rho} \cdot P \cdot \rho: \widehat{W}_{0, b-1}^{1,2} \rightarrow L_{0, b}^{2}$ has the same index as $P$. Noting

$$
P_{\rho}=\left\{\begin{array}{cc}
\frac{\partial}{\partial t}-(B-\beta I d) & \text { on End } \\
P & \text { on End }
\end{array}\right.
$$

then Theorem 5.2 says the following for $P_{\rho}$.

$$
\text { index }\left.P_{\rho}\right|_{\widehat{W}_{0, b-1}^{1,2} \rightarrow L_{0, b}^{2}}=\int_{X} \alpha_{0} d v o l-\frac{E t a(B-\beta I d)}{2}-\frac{E t a(-B)}{2}-\frac{d_{\beta}}{2}-\frac{d_{0}}{2} .
$$

The equality between (55) and (56) yields the desired identity. 


\section{Applications}

Definition 6.1. Let $\Gamma$ be a section or connection of $E \rightarrow C y l_{0}$. Suppose $\Gamma$ satisfies an elliptic equation of order $m_{0}$. For any $C_{0}>0, \tau \geq 0$, and real number $b$, we say that $\Gamma$ converges to $\Gamma_{0}$ (at least) at the rate $\frac{C_{0} e^{-\tau t}}{t^{b}}\left[\right.$ or $\left.O\left(\frac{e^{-\tau t}}{t^{b}}\right)\right]$, if $\Gamma_{0}$ is a section or connection of $E \rightarrow Y$ respectively and $\lim \sup _{t \rightarrow \infty} \frac{e^{-\tau t}}{t^{b}}\left|\Gamma-\Gamma_{0}\right|_{C^{m_{0}, \alpha}\left(S_{t}\right)}<C_{0}($ or $<\infty)$ respectively.

When $\tau>0$, We say that the convergence is exponential. When $\tau=0$, We say that it's polynomial. We only prove 1.11 in full detail, 1.9 follows similarly.

\subsection{Yang-Mills connections: proof for Corollary 1.11}

Denoting $A-A_{O}$ by $a$, the YM-equation is $0=d_{A}^{\star} F_{A}=d_{A_{O}+a}^{\star}\left(d_{A_{O}} a+F_{A_{O}}+\frac{1}{2}[a, a]\right)$.

Thus $\Delta_{A_{O}, \text { Hodge }} a+(-1)^{n+1} \star\left[a, \star F_{A_{O}}\right]=Q_{Y M}(a)-d_{A_{O}}^{\star} F_{A_{O}}$ assuming $d_{A_{O}}^{\star} a=0$,

where $\Delta_{A_{O}, \text { Hodge }}=d_{A_{O}}^{\star} d_{A_{O}}+d_{A_{O}} d_{A_{O}}^{\star}$ is the Hodge Laplacian, and

$$
Q_{Y M}(a)=-\frac{d_{A_{O}}^{\star}[a, a]}{2}+(-1)^{n} \star\left[a, \star d_{A_{O}} a\right]+\frac{(-1)^{n}}{2} \star[a, \star[a, a]] .
$$

We note $Q_{Y M}$ is quadratic in $a$. We routinely verify

$(-1)^{n+1} \star\left[a, \star F_{A_{O}}\right]=F_{A_{O}} \underline{\otimes} a\left(\left[15\right.\right.$, Definition 3.2]), $\Delta_{A_{O}, \text { Hodge, } g_{E}}=\nabla_{A_{O}}^{\star_{O}} \nabla_{A_{O}}+F_{A_{O}} \underline{\otimes}_{g_{E}} a$.

Then in cylindrical coordinates $\left(r=e^{-t}\right)$, let $a=v d t+\theta(\theta$ does not contain $d t)$, we find

$$
\begin{aligned}
& \Delta_{A_{O}, \text { Hodge }, E} a+(-1)^{n+1} \star_{E}\left[a, \star_{E} F_{A_{O}}\right]=\nabla_{A_{O}}^{\star_{E}} \nabla_{A_{O}}+2 F_{A_{O}} \underline{\otimes}_{g_{E}} a \\
& =\quad-e^{2 t}\left|\begin{array}{cc}
d t & 0 \\
0 & I d
\end{array}\right|\left\{\frac{\partial^{2}}{\partial t^{2}}-(n-4) \frac{\partial}{\partial t}-B\right\}\left[\begin{array}{c}
v \\
\theta
\end{array}\right] \text {. Let } Y=\mathbb{S}^{n-1}, B \text { is }
\end{aligned}
$$

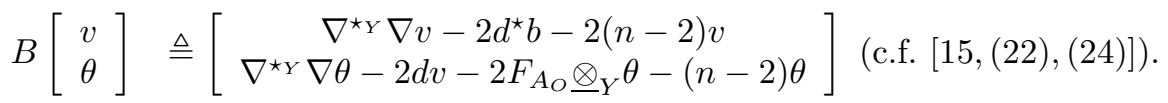

As an usual strategy for non-linear equations, we view $Q_{Y M}$ as a linear operator defining

$$
\widehat{Q}_{Y M, a}(b)=-\frac{d_{A_{O}}^{\star}[b, a]}{2}+(-1)^{n} \star\left[b, \star d_{A_{O}} a\right]+\frac{(-1)^{n}}{2} \star[b, \star[a, a]] .
$$

Hence $Q_{Y M}(a)=\widehat{Q}_{Y M, a}(a)$, and we can write (57) in cylindrical coordinates as

$$
P_{Y M}(a) \triangleq\left\{e^{-2 t}\left(\Delta_{A_{O}, \text { Hodge }}+(-1)^{n+1} \star\left[\cdot, \star F_{A_{O}}\right]\right)-e^{-2 t} \widehat{Q}_{Y M, a}\right\} a=-e^{-2 t} d_{A_{O}} F_{A_{O}} .
$$

The conditions on $g, a$, (59), and (60) implies $P_{Y M}$ is ATID in the cylindrical coordinates. Moreover, that $d_{A_{O}}^{\star_{E}} F_{A_{O}}=0$ (tangent connection is Yang-Mills) implies $-e^{-2 t} d_{A_{O}}^{\star} F_{A_{O}} \in$ $C_{1,0}^{\alpha}\left(\overline{C y l}_{-\log R}\right)$ (exponential decay). Applying $Q_{0,+}^{P_{Y M},-2 \log R}\left(=Q_{\underline{0},-}^{P_{Y M},-2 \log R}\right)$ to both sides of (62), Lemma 4.4 III implies that a decays exponentially. This in turn means $P_{Y M}$ satisfies $S(l)$ for all $l>1$. Then Corollary $1.11 I$ follows from applying Proposition 4.5 to $a+Q_{0,+}^{P_{Y M},-2 \log R} e^{-2 t} d_{A_{O}}^{\star} F_{A_{O}} \in \operatorname{Ker} P_{Y M}$ and Theorem 3.3 to $Q_{0,+}^{P_{Y M},-2 \log R} e^{-2 t} d_{A_{O}}^{\star} F_{A_{O}}$.

Combining $I$ and Lemma 7.2 , the proof of $I I$ is complete.

\subsection{Minimal Surfaces: proof for Corollary 1.9}

By [1, Section 5, page 247], in the cylindrical coordinate $t=\log \frac{1}{|x|}$, the graph type minimal sub-manifold equation can be written as (63) in terms of a section $u$ to $\left.T^{\perp} \Sigma\right|_{\mathbb{S}^{N}}$ (the normal bundle of $\Sigma$ in $\mathbb{S}^{N}$ ). We note (the transition functions of) $\left.T^{\perp} \Sigma\right|_{\mathbb{S}^{N}}$ does not depend on $t$ (or $|x|$ ), then this bundle is in the case considered by Definition 1.1.

$$
P_{M S M} u \triangleq L u+\mathfrak{R}(u)+\left(M_{\Sigma}-L_{\Sigma}\right) u=0, \text { where }
$$


- $L u=u^{\prime \prime}-(n+1) u^{\prime}+L_{\Sigma} u$ (see [16, Page 565] and [1, (1.8)]),

- $\mathfrak{R}(u)$ satisfies [16, (1.9)], $L_{\Sigma}$ is the linearisation of $M_{\Sigma}$ (see [1, (5.2) and Page 248].

By the idea in (61), we can view $\mathfrak{R}+M_{\Sigma}-L_{\Sigma}$ as a linear operator i.e.

$$
\begin{aligned}
\left(\Re+M_{\Sigma}-L_{\Sigma}\right)(v) \triangleq Q_{M S M}(v)= & a\left(x, t, u, \nabla u, \nabla^{2} u, u^{\prime}\right) \cdot \nabla^{2} v+b\left(x, t, u, \nabla u, \nabla^{2} u\right) \cdot v^{\prime} \\
& +c\left(x, t, u, \nabla u, \nabla^{2} u\right) \cdot \nabla v^{\prime}+d\left(x, t, u, \nabla u, \nabla^{2} u\right) \cdot v^{\prime \prime}
\end{aligned}
$$

where $a, b, c, \nabla$ can be found in [16, the paragraph enclosing (1.9); between line 1 in page 565 and (7.35)]. Exactly as the proof of Corollary[1.11 $u$ decays as $\frac{\delta_{0}}{t}$ implies $P_{M S M}$ is 0-ATID. Thus Proposition 4.5 [and the discussion below [621)] yields the desired improvement.

\section{Appendix}

Proof of Theorem 3.2 (i) for second-order operators: We mainly focus on the case when $a_{1}-2 \beta>0$. We solve the second-order ODE in (11) according to the following.

\begin{tabular}{|l|l|l|l|}
\hline & $\begin{array}{l}m \triangleq a_{1}-2 \beta . \\
\text { Assume } m>0 .\end{array}$ & $\begin{array}{l}\text { Corresponding solution } u_{\lambda} \triangleq \dot{Q}_{\lambda}^{P_{\beta}^{0}} f_{\lambda} \text { and the derivative. } \\
\text { Det } \triangleq m^{2}+4 \lambda, \quad \mu \triangleq \frac{\sqrt{|D e t|}}{2}, \mu^{+} \triangleq \frac{m}{2}+\mu, \mu^{-} \triangleq \frac{m}{2}-\mu\end{array}$ \\
\hline 1 & Det $>0, \lambda \neq 0$ & $\begin{array}{l}u_{\lambda}=-\frac{1}{\sqrt{D e t}}\left[e^{\mu^{+}} t \int_{t}^{\infty} e^{-\mu^{+} s} f_{\lambda} d s+e^{\mu^{-} t} \int_{1}^{t} e^{-\mu^{-} s} f_{\lambda} d s\right], u_{\lambda}^{\prime}= \\
-\frac{1}{\sqrt{D e t}}\left[\mu^{+} e^{\mu^{+} t} \int_{t}^{\infty} e^{-\mu^{+} s} f_{\lambda} d s+\mu^{-} e^{\mu^{-} t} \int_{1}^{t} e^{-\mu^{-} s} f_{\lambda} d s\right]\end{array}$ \\
\hline 2 & $\lambda=0, b>\frac{1}{2}$ & $\begin{array}{l}u_{\lambda}=\frac{1}{\sqrt{D e t}}\left\{-e^{\mu^{+} t} \int_{t}^{\infty} e^{-\mu^{+} s} f_{\lambda} d s+\int_{t}^{\infty} f_{\lambda} d s\right\}, \quad u_{\lambda}^{\prime} \\
-\frac{\mu^{+}}{\sqrt{D e t}} e^{\mu^{+} t} \int_{t}^{\infty} e^{-\mu^{+} s} f_{\lambda} d s\end{array}$ \\
\hline 3 & $\lambda=0, b<\frac{1}{2}$ & $\begin{array}{l}u_{\lambda}=\frac{1}{\sqrt{D e t}}\left\{-e^{\mu^{+} t} \int_{t}^{\infty} e^{-\mu^{+} s} f_{\lambda} d s-\int_{1}^{t} f_{\lambda} d s\right\}, \quad u_{\lambda}^{\prime} \quad \\
-\frac{\mu^{+}}{\sqrt{D e t}} e^{\mu^{t} t} \int_{t}^{\infty} e^{-\mu^{+} s} f_{\lambda} d s\end{array}$ \\
\hline 4 & Det $=0$ & $u_{\lambda}=e^{\frac{m t}{2}} \int_{t}^{\infty} e^{-\frac{m s}{2}} f_{\lambda}(s-t) d s$ & \\
\hline 5 & $D e t<0$ & $u_{\lambda}=\frac{e^{\frac{m t}{2}}}{\mu}\left\{\int_{t}^{\infty} e^{-\frac{m s}{2}} f_{\lambda}[\cos \mu t \sin (\mu s)-\sin \mu t \cos (\mu s)] d s\right\}$ \\
\hline
\end{tabular}

Case 1, 5 in (64): When $\lambda \neq 0$, we have $\frac{\sqrt{|\lambda|}}{C} \leq \mu^{+} \leq C \sqrt{|\lambda|},-\frac{\sqrt{|\lambda|}}{C} \leq \mu^{-} \leq-C \sqrt{|\lambda|}$. We use (25), (26) to estimate $u_{\lambda}$ and $u_{\lambda}^{\prime}$ termwise, then use the second-order equation in (11) to estimate $u_{\lambda}^{\prime \prime}$. Then we obtain the following for Case 1 and 4.

$$
\int_{1}^{\infty}\left|u_{\lambda}^{\prime \prime}\right|^{2} t^{2 \gamma} d t+|\lambda| \int_{1}^{\infty}\left|u_{\lambda}^{\prime}\right|^{2} t^{2 \gamma} d t+\lambda^{2} \int_{1}^{\infty} u_{\lambda}^{2} t^{2 \gamma} d t \leq C \int_{1}^{\infty} f_{\lambda}^{2} t^{2 \gamma} d t
$$

Since $|\cos x|,|\sin x| \leq 1$ for all $x$, and $\frac{1}{\sqrt{\lambda}} \leq C$ when $\lambda \neq 0[\operatorname{Spec}(B)$ is discrete], (65) holds for Case 5 in similar way.

Cases 2,3 in (64): We still do the termwise estimates. Using (25) for $e^{\mu^{+} t} \int_{t}^{\infty} e^{-\mu^{+} s} f_{\lambda} d s$, (26) for $e^{-\mu^{+} t} \int_{1}^{t} e^{\mu^{+} s} f_{\lambda} d s$, and (23) for $\int_{t}^{\infty} f_{\lambda} d s$, we obtain

$$
\int_{1}^{\infty}\left|u_{\lambda}^{\prime \prime}\right|^{2} t^{2 b} d t+\int_{1}^{\infty}\left|u_{\lambda}^{\prime}\right|^{2} t^{2 b} d t+\int_{1}^{\infty} u_{\lambda}^{2} t^{2 b-2} d t \leq C \int_{1}^{\infty} f_{\lambda}^{2} t^{2 b} d t
$$

Remark 2.5. (65), (66) amount to $\left|\widetilde{Q}_{0, b}^{P_{\beta}^{0}} f\right|_{\widehat{W}_{0, \gamma, b-1}^{2,2}\left(C y l_{1}\right)} \leq C|f|_{L_{0, \gamma, b}^{2}\left(C y l_{1}\right)}$. The argument between (31) and (33) gives the desired local right inverse $Q_{\beta, b}^{P^{0}, t_{0}}$ with the desired bound.

The proof when $a_{1}-2 \beta<0$ is by the same analysis, but to the following table of solutions [using the definitions in the first row of (64) but assuming $m=a_{1}-2 \beta<0$ ].

\begin{tabular}{|l|l|l|}
\hline 1 & Det $>0, \lambda \neq 0$ & the same as Case 1 in (64) \\
\hline 2 & $\lambda=0, b>\frac{1}{2}$ & $u_{\lambda}=\frac{1}{\sqrt{D e t}}\left\{-\int_{t}^{\infty} f_{\lambda} d s-e^{\mu_{-} t} \int_{1}^{t} e^{-\mu_{-} t} f_{\lambda} d s\right\}$ \\
\hline 3 & $\lambda=0, b<\frac{1}{2}$ & $u_{\lambda}=\frac{1}{\sqrt{D e t}}\left\{-e^{\mu^{-} t} \int_{1}^{t} e^{-\mu^{-} s} f_{\lambda} d s+\int_{1}^{t} f_{\lambda} d s\right\}$ \\
\hline 4 & $D e t=0$ & $u_{\lambda}=e^{\frac{m t}{2}} \int_{1}^{t} e^{-\frac{m s}{2}} f_{\lambda}(t-s) d s$ \\
\hline 5 & $D e t<0$ & $u_{\lambda}=-\frac{e^{\frac{m t}{2}}}{\mu}\left\{\int_{1}^{t} e^{-\frac{m s}{2}} f_{\lambda}[\cos \mu t \sin (\mu s)-\sin \mu t \cos (\mu s)] d s\right\}$ \\
\hline
\end{tabular}


The proof when $m=a_{1}-2 \beta=0$ and $\lambda>0$ is much easier: we only need to use (exactly) the solutions in Case 1 of (67). Case 2-5 are super-indicial (which we don't consider).

In the cone setting as Corollary 1.11, let $\nabla_{A_{O}}^{\star} \nabla_{A_{O}}$ denote the rough Laplacian on $\Omega^{0}(a d E)$. By [15, (17)], in cylindrical coordinate $t=-\log r$,

$$
\nabla_{A_{O}}^{\star} \nabla_{A_{O}} \chi=e^{2 t}\left\{\frac{d^{2} \chi}{d t^{2}}-(n-2) \frac{d \chi}{d t}+\Delta_{A_{O}, \mathbb{S}^{n-1}} \chi\right\},
$$

where $\Delta_{A_{O}, \mathbb{S}^{n-1}}$ is the rough Laplacian on the link. Let $C_{\gamma, b, c o n e}^{k, \alpha}$ denote the weighted Schauder-spaces in the cone setting defined in [15, Definition 2.10]. On 0 -forms, we note that pulling back is an isomorphism $C_{\gamma, b, c o n e}^{k, \alpha}\left[B_{O}(R)\right] \rightarrow C_{-\gamma, b}^{k, \alpha}\left(C y l_{-\log R}\right)$.

Definition 7.1. We say that $A_{O}$ is irreducible if every parallel section to $\Omega^{0}(a d E)$ is 0 everywhere. This means 0 is not an eigenvalue of $\Delta_{A_{O}, \mathbb{S}^{n-1}}$.

Lemma 7.2. (Existence of Coulomb gauge) Under the setting in the first paragraph of Corollary 1.11 (for any $n \geq 3$ ), suppose $A_{O}$ is irreducible. Suppose $A$ is a $C^{2}-$ connection on $B_{O}(R) \backslash O$ such that

$$
\left|A-A_{O}\right|_{\left.C_{1, b, c o n e}^{1, \alpha}\left[B_{O}(R)\right]\right)}<\delta_{0} \text { for some } b \geq 0 .
$$

Then there exists a gauge $s$ on $B_{O}(R)$ such that

- $|s-I d|_{C_{0, b, c o n e}^{2, \alpha}\left[B_{O}(R)\right]} \leq C\left|A-A_{O}\right|_{\left.C_{1, b, c o n e}^{1, \alpha}\left[B_{O}(R)\right]\right)} \leq C \delta_{0}$,

- $d_{A_{O}}^{\star}\left[s(A)-A_{O}\right]=0$ in a smaller (truncated) ball $B_{O}\left(R^{\prime}\right) \backslash O$, and

$$
\left|S(A)-A_{O}\right|_{\left.C_{1, b, c o n e}^{1, \alpha}\left[B_{O}\left(R^{\prime}\right)-O\right]\right)} \leq C\left|A-A_{O}\right|_{\left.C_{1, b, c o n e}^{1, \alpha}\left[B_{O}(R)-O\right]\right)} \leq C \delta_{0} .
$$

Proof. Let $a \triangleq A-A_{O}$. By Theorem 3.3 and paragraph enclosing (68), for any $b \geq 0$ and $R^{\prime}$ small enough, $\nabla_{A}^{\star} \nabla_{A}$ is invertible: $\left.\left.C_{0, b, c o n e}^{2, \alpha}\left[B\left(R^{\prime}\right)\right]\right|_{\Omega^{0}(a d E)} \longrightarrow C_{2, b, \text { cone }}^{\alpha}\left[B\left(R^{\prime}\right)\right]\right|_{\Omega^{0}(\text { adE })}$. Writing $s=e^{-\chi}$, then

$$
d_{A_{O}}^{\star}\left[s(A)-A_{O}\right]=d_{A_{O}}^{\star}\left[s^{-1} a s+s^{-1} d_{A_{O}} s\right]=d_{A_{O}}^{\star}\left[e^{-\chi} a e^{\chi}+e^{-\chi} d_{A_{O}} e^{\chi}\right]
$$

is a continuously differentiable map:

$$
\left.C_{1, b, c o n e}^{1, \alpha}\left[B_{O}(R)\right]\right|_{\Omega^{1}(a d E)} \times\left.\left. C_{0, b, c o n e}^{2, \alpha}\left[B_{O}(R)\right]\right|_{\Omega^{0}(a d E)} \longrightarrow C_{2, b, c o n e}^{\alpha}\left[B_{O}(R)\right]\right|_{\Omega^{0}(a d E)} .
$$

Then the proof is complete by the standard argument in [6, Proposition 2.3.4].

Proof of (25), (26): For any $s \geq \frac{1}{10}$, leaving the proof to the readers, we have

$$
\left|\int_{1}^{s} e^{\mu t} t^{d} d t\right| \leq C_{l_{\mu}, d} \frac{e^{\mu s} s^{d}}{\mu} \text { when } \mu>0, \int_{s}^{\infty} e^{\mu t} t^{d} d t \leq C_{l_{\mu}, d} \frac{e^{\mu s} s^{d}}{-\mu} \text { when } \mu<0 .
$$

For (25), Hölder's inequality and the change of variable $z=s-t$ yield

$$
\begin{aligned}
& \left(\int_{t}^{\infty} e^{-\mu s} f(s-t)^{\vartheta} d s\right)^{p} \leq\left(\int_{t}^{\infty} e^{-\mu s} f^{p} d s\right)\left(\int_{t}^{\infty} e^{-\mu s}(s-t)^{\frac{p \vartheta}{p-1}} d s\right)^{p-1} \\
\leq & \left.C\left(\int_{t}^{\infty} e^{-\mu s} f^{p} d s\right) \frac{e^{-\mu(p-1) t}}{\mu^{\vartheta p+p-1}}\right) .
\end{aligned}
$$

Then (72) and (73) yield

$$
\begin{aligned}
& \int_{\frac{1}{10}}^{\infty}\left(e^{\mu t} t^{b} \int_{t}^{\infty} e^{-\mu s} f(s-t)^{\vartheta} d s\right)^{p} d t \leq \frac{1}{\mu^{p \vartheta+p-1}}\left(\int_{\frac{1}{10}}^{\infty} e^{-\mu s} f^{p} d s\right)\left(\int_{\frac{1}{10}}^{s} e^{\mu t} t^{p b} d t\right) \\
\leq & \frac{C_{l_{\mu}, p, b}}{\mu^{p(1+\gamma)}} \int_{0}^{\infty} f^{p} s^{p b} d s . \quad \text { The proof of (25) is complete. }
\end{aligned}
$$

The proof of (26) is similar. 
Proof of Remark 1.8: We only have to show the "only if". Without loss of generality, we only consider the Schauder theory, and assume $m_{0}=1, \gamma=b=1, \vec{\beta}=0, \sigma_{1}=\sigma_{2}=I d$. For any $\phi_{0} \in \operatorname{Ker} B_{P^{0}}$, let $f=\frac{\phi_{0}}{t}$. When $P=\frac{\partial}{\partial t}-B$ (translation-invariant on the end) and $t \geq 10$, the general solution to $P u=\frac{\phi_{0}}{t}$ is $(\log t+C) \phi_{0} \notin \widehat{C}_{0,0}^{1, \alpha}$. Hence any extension $\widetilde{f}$ of $f$ to the whole $N$ is not in Range $\left.P\right|_{\widehat{C}_{0,0}^{1, \alpha}}$.

On the other hand, let $f_{k}=\frac{\phi_{0}}{t^{1+\frac{1}{k}}}$, Theorem 3.2 implies each $f_{k}$ admits an extension $\widetilde{f}_{k} \in$ Range $\left.P\right|_{\widehat{C}_{0,0}^{1, \alpha}}$, and $\widetilde{f}_{k}$ tends to $\widetilde{f} \in C_{0,1}^{\alpha}$ which extends $f$. Then RangeP$\left.\right|_{\widehat{C}_{0,0}^{1, \alpha}}$ is not closed in $C_{0,1}^{\alpha}$.

\section{References}

[1] D. Adam, L.Simon. Rates of Asymptotic Convergence Near Isolated Singularities of Geometric Extrema. Indiana Univ. Math. J. 37 (1988), no. 2, 225-254.

[2] C. Amrouche, V. Girault, J. Giroire. Dirichlet and neumann exterior problems for the $n$ dimensional Laplace operator an approach in weighted Sobolev spaces. Journal de Mathmatiques Pures et Appliques Volume 76, Issue 1, January 1997, 55-81.

[3] M.F. Atiyah, V.K. Patodi, I.M. Singer. Spectral asymmetry and Riemannian Geometry. I. Math. Proc. Camb. Phil. Soc. 1975.

[4] E. Calabi, Extremal Kähler metrics. Seminar on Differential Geometry, volume 16 of 102, 259290. Ann. of Math. Studies, University Press, 1982.

[5] S.Y. A. Chang, P. C. Yang. Non-linear Partial Differential Equations in Conformal Geometry ICM 2002. Vol. I.189-207.

[6] S.K. Donaldson, P.B. Kronheimer. The geometry of Four-Manifolds. Oxford Mathematical Monographs. 1990.

[7] L.C. Evans. Partial differential equations. Graduate Studies in Mathematics, Vol 19. AMS.

[8] D. Gilbarg, N.S. Trudinger. Elliptic Partial Differential Equations of Second Order. Springer.

[9] G.H. Hardy, J.E. Littlewood, G. Pólya. Inequalities. second edition, Cambridge, 1952.

[10] R.B. Lockhart, R.C. McOwen. On elliptic systems in $\mathbb{R}^{n}$. Acta Math. 150 (1983), 125-135.

[11] R.B. Lockhart, R.C. McOwen. Elliptic differential operators on noncompact manifolds. Annali della Scuola Normale Superiore di Pisa - Classe di Scienze (1985) Volume 12, Issue 3, 409-447.

[12] R. Mazzeo. Elliptic theory of differential edge operators I. Comm. Partial Differential Equations 16 (1991), no.10, 1615-1664.

[13] V.G. Maz'ya, B.A. Plamenevskii. Estimates in $L_{p}$ and in Hölder Classes and the MirandaAgmon Maximum Principle for Solutions of Elliptic Boundary Value Problems in Domains with Singular Points on the Boundary. Translation of Math. Nachr. 81 (1978), 25-82.

[14] R.B. Melrose, G. Mendoza. Elliptic operators of totally characteristic type. MSRI, Berkeley, CA June 1983. MSRI 047-83.

[15] Y.Q. Wang. Deformation of singular connections I: $G_{2}$-instantons with point singularities. arXiv:1602.05701.

[16] L. Simon. Asymptotics for a class of non-linear evolution equations, with applications to geometric problems. Annals of Mathematics, 118 (1983), 525-571.

[17] K. Yosida. Functional analysis. Springer Classics in Mathematics. Second edition.

[18] G.Q. Zhang, Y.Q. Lin. Lecture Notes on Functional Analysis, Vol. 1 (Mandarin Chinese). Peking University Press; 1st edition. March 1, 1987. 